\title{
Inverse Optimal Attitude Stabilization of Flexible Spacecraft with Actuator Saturation
}

\author{
Chutiphon Pukdeboon \\ Nonlinear Dynamic Analysis Research Center, Department of Mathematics, Faculty of Applied Science, King Mongkut's University of \\ Technology North Bangkok, Bangkok 10800, Thailand \\ Correspondence should be addressed to Chutiphon Pukdeboon; cpd@kmutnb.ac.th
}

Received 28 December 2015; Accepted 28 March 2016

Academic Editor: Kenneth M. Sobel

Copyright (C) 2016 Chutiphon Pukdeboon. This is an open access article distributed under the Creative Commons Attribution License, which permits unrestricted use, distribution, and reproduction in any medium, provided the original work is properly cited.

\begin{abstract}
This paper presents a new robust inverse optimal control strategy for flexible spacecraft attitude maneuvers in the presence of external disturbances and actuator constraint. A new constrained attitude controller for flexible spacecraft is designed based on the Sontag-type formula and a control Lyapunov function. This control law optimizes a meaningful cost functional and the stability of the resulting closed-loop system is ensured by the Lyapunov framework. A sliding mode disturbance observer is used to compensate unknown bounded external disturbances. The ultimate boundedness of estimation error dynamics is guaranteed via a rigorous Lyapunov analysis. Simulation results are provided to demonstrate the performance of the proposed control law.
\end{abstract}

\section{Introduction}

Attitude control of flexible spacecraft has been an important problem in many developments of spacecraft technology. Recent spacecraft requires the structure of a rigid hub with flexible appendages, such as antenna and solar array. Because of the strong coupling between the hub and flexible appendages, the vibration of flexible appendages will be induced. This may lead to performance degradation or instability if the vibration is not suppressed as rapid as possible. In addition, owing to the environment and measurement factors, the external torque disturbance cannot be avoided. Inertia matrix uncertainty and external disturbance are required to be considered by researcher in attitude controller designs of a spacecraft. Recently, great attention has been paid to the vibration suppression and robust attitude controller designs for flexible spacecraft such as passive control [1], input shaping [2], sliding mode control [3, 4], and active disturbance rejection control $[5,6]$.

Optimal fuel consumption and time optimal control problems for spacecraft attitude maneuvers are very practical and important issues. The synthesis of optimal attitude control law for spacecraft attitude maneuvers has become increasingly popular among researchers. In [7] the timevarying linear quadratic regulator (LQR) method was applied to a nonlinear control problem but the approximation of spacecraft system was required to meet the optimality and stability conditions. In $[8,9]$ state-dependent Riccati equation (SDRE) techniques were successfully used to develop optimal controller for spacecraft attitude maneuvers. In Xin and Balakrishnan [10], SDRE and neural network schemes were merged to develop a robust optimal attitude control law for spacecraft under an uncertain moment of inertia. However, the SDRE method requires to solve the Riccati equation repetitively at every integration step. It may be difficult to use the SDRE method if the system order is higher. Xin and Pan [11] have applied the $\theta-D$ technique to design a nonlinear integrated position and attitude suboptimal control law. An inverse optimal control approach was presented in [12] to construct the optimal controller for regulation of the rigid body. Recently, attitude controller designs for rigid spacecraft using inverse optimal control schemes have been developed (see, e.g., $[13,14])$. The inverse optimal control approach avoids the task of solving a HamiltonJacobi-Bellman equation and offers a globally asymptotically stabilizing control law which is optimal with respect to 
a meaningful cost functional. Using this method, a stabilizing control law can be designed using Sontag's formula [15] or Freeman's formula [16] with directional information supplied by control Lyapunov function (CLF) $[17,18]$.

As extensions of the above studies, optimal control and robust control have been merged to obtain robust optimal control laws. Various methods for developing robust optimal controllers for the attitude control of a rigid spacecraft have been proposed in the literature. Nonlinear $H_{\infty}$ control strategies were proposed in $[19,20]$ to develop stabilizing feedback controllers for the spacecraft tracking motion. Later, the inverse optimal control method was used to develop attitude controllers for a rigid spacecraft in [21]. Luo et al. [22] developed an $H_{\infty}$ inverse optimal adaptive controller for attitude tracking of spacecraft. Adaptive control and nonlinear $\mathrm{H}_{\infty}$ control have also been merged to design robust optimal controllers. Zheng and $\mathrm{Wu}$ [23] proposed nonlinear $H_{\infty}$ controller designs for spacecraft and a $\gamma$-level disturbance attenuation could be attained. Pukdeboon and Zinober [24] developed robust optimal control laws based on the optimal sliding mode control technique for attitude tracking of spacecraft. Optimal sliding mode control and inverse optimal control schemes for flexible spacecraft attitude maneuvers have been designed in [25] and [5], respectively. However, these control methods did not consider the effect of actuator saturation. In practical situation, due to the physical restriction and energy consumption, if actuator saturation is not handled effectively, a performance degradation or system instability may occur. Moreover, control methods mentioned above may lead to the unwinding phenomenon encountered in unit quaternion based attitude systems since these control laws consider only one of two equilibrium points of unit quaternion [26].

The main contributions of this paper are as follows:

(I) A robust inverse optimal control method for flexible spacecraft attitude maneuvers in the presence of actuator constraint is proposed in this paper. This controller can prevent the unwinding phenomenon.

(II) A new inverse optimal control problem for flexible spacecraft attitude maneuvers with input saturation is studied. The proper control Lyapunov function is chosen and then used to solve this problem. Finding a control Lyapunov function and optimal feedback control to solve the inverse optimal control problem for the flexible spacecraft attitude control system is very difficult and has not been previously examined.

(III) A new sliding mode disturbance observer is developed and combined with the proposed attitude controller. The uniformly ultimate boundedness stability of the proposed disturbance observer is guaranteed via a rigorous Lyapunov analysis.

This paper is organized as follows. Section 2 introduces some preliminary results which are required for the following discussion in this paper. In Section 3 the dynamic equations and attitude kinematics of a flexible spacecraft $[27,28]$ are described. Also, the control objective is provided. Section 4 proposes an inverse optimal control design with input saturation. The proposed CLF is selected to solve the inverse optimal control problem of flexible spacecraft and then an optimal stabilizing controller is designed. In Section 5, a sliding mode disturbance observer is designed and then used to develop a robust optimal attitude controller. The ultimate boundedness of estimation errors is guaranteed using the Lyapunov stability theory. In Section 6 an example of spacecraft attitude manoeuvres is presented to illustrate the performance of the developed control law. In Section 7 we present conclusions.

\section{Mathematical Preliminaries}

2.1. Inverse Optimal Control via Control Lyapunov Function. Basic concepts in the realm of nonlinear stabilization are given below. We consider the nonlinear dynamic system

$$
\dot{x}=f(x)+g(x) u,
$$

where $x \in \mathscr{R}^{n}$ denotes the system state, $u \in \mathscr{R}^{m}$ is the control vector, $f: \mathscr{R}^{n} \rightarrow \mathscr{R}^{n}$ and $g: \mathscr{R}^{n} \rightarrow \mathscr{R}^{n \times m}$ are continuous functions, respectively, with $f(0)=0$.

Based on the definition of control Lyapunov function (CLF) in $[17,29]$, we provide the following definition.

Definition 1 (see $[17,29]$ ). A differentiable, positive definite and radially unbounded function $V(x): \mathscr{R}^{n} \rightarrow R^{+}$is called a control Lyapunov function (CLF) of system (29) if, for each $x \neq 0$,

$$
L_{g} V=0 \Longrightarrow L_{f} V<0 \text {, }
$$

where the functions $L_{f} V$ and $L_{g} V$ denote the Lie derivatives of the Lyapunov function $V(x)$ with respect to the vector fields defining the system. These functions are defined as $L_{f} V=[\partial V / \partial x]^{T} f(x)$ and $L_{g} V=[\partial V / \partial x]^{T} g(x)$.

We next consider a globally stabilizing control law

$$
u(x)=\kappa^{*}(x)=-R^{-1}(x)\left(L_{g} V(x)\right)^{T},
$$

where $R(x)$ is a symmetric positive definite matrix. When a CLF is known for system (1), an inverse optimal controller can be designed using the following lemma.

Lemma 2 (see [29]). The globally stabilizing control law solves system (1) with respect to the cost functional

$$
\inf _{u} \int_{0}^{\infty}\left(l(x)+u^{T} R u\right) d t
$$

wherel $l(x)=-L_{f} V(x)-(1 / 2) L_{g} V(x) \kappa^{*}(x) \geq 0$.

If $V(x)$ is a positive definite, radially unbounded function such that $\dot{V}(x) \leq 0$ is achieved with the control law $u=$ $(1 / 2) \kappa^{*}=-(1 / 2) R^{-1}\left(L_{g} V(x)\right)^{T}$, it follows that

$$
\dot{V}=L_{f} V(x)+\frac{1}{2} L_{g} V(x) \kappa^{*}(x) \leq 0 .
$$


When the function $l(x)$ is set to be $-\dot{V}$, one obtains

$$
l(x)=-L_{f} V(x)-\frac{1}{2} L_{g} V(x) \kappa^{*}(x) \geq 0,
$$

and $V(x)$ becomes a solution of the Hamilton-Jacobi-Bellman (HJB) equation

$$
l(x)+L_{f} V(x)-\frac{1}{4} L_{g} V R^{-1}\left(L_{g} V\right)^{T}=0 .
$$

According to the standard result of optimal control theory, the control law (3) is optimal among all $u(t)$ that globally asymptotically stabilize system (1).

Remark 3. In the inverse optimal approach, a globally stabilizing feedback control law $u(x)$ is designed first and then it is required to find $l(x) \geq 0$ and $R(x)>0$ such that $u$ optimizes (4). The problem is inverse because the functions $l(x)$ and $R(x)$ are a posteriori found by the stabilizing feedback law, rather than a priori selected by the designer.

\section{Flexible Spacecraft Attitude Model}

3.1. Kinematics of Flexible Spacecraft. In this paper the unit quaternion is used to represent the attitude system of a flexible spacecraft. We define the rigid body attitude and angular velocity of the rigid spacecraft body frame with respect to the inertia axis frame as $\bar{q}=\left[\begin{array}{ll}q_{0} & q^{T}\end{array}\right]^{T} \in \mathscr{R} \times \mathscr{R}^{3}$ and $\omega \in \mathscr{R}^{3}$, respectively. Then, the kinematics in terms of the quaternion is given by [27]

$$
\dot{\bar{q}}=\frac{1}{2}\left[\begin{array}{c}
-q^{T} \\
T(\bar{q})
\end{array}\right] \omega,
$$

where $T(\bar{q})=q^{\times}+q_{0} I_{3}$ with $I_{3}$ the $3 \times 3$ identity matrix. Here, notation $q^{\times}$denotes the skew-symmetric matrix which is defined as

$$
q^{\times}=\left[\begin{array}{ccc}
0 & -q_{3} & q_{2} \\
q_{3} & 0 & -q_{1} \\
-q_{2} & q_{1} & 0
\end{array}\right] .
$$

The components of $\bar{q}$ satisfy the condition

$$
\|\bar{q}\|=q_{0}^{2}+q^{T} q=1,
$$

where $\|\cdot\|$ is the Euclidean norm. A more detailed explanation of the quaternion and other attitude representations are presented in $[27,30]$.

3.2. Dynamics of Flexible Spacecraft. The dynamics of a flexible spacecraft is governed by [28]

$$
\begin{aligned}
J \dot{\omega}+\delta^{T} \ddot{\eta} & =-\omega^{\times}\left(J \omega+\delta^{T} \dot{\eta}\right)+u+d \\
\ddot{\eta}+K \dot{\eta}+C \eta & =-\delta \dot{\omega},
\end{aligned}
$$

where $J \in \mathscr{R}^{3 \times 3}$ is the symmetric inertia matrix of the whole spacecraft, $\eta \in \mathscr{R}^{n}$ is the modal displacement vector with $n$ being the mode number, and $\delta \in \mathscr{R}^{n \times 3}$ is the coupling matrix between the central rigid body and the flexible attachments. $u \in \mathscr{R}^{3}$ denotes the control torque vector, $d \in \mathscr{R}^{3}$ represents the external disturbance torque, and $K$ and $C$ denote the stiffness and damping matrices, respectively, which are defined as

$$
\begin{aligned}
& K=\operatorname{diag}\left(2 \zeta_{i} \omega_{n i}, \quad i=1,2, \ldots, N\right), \\
& C=\operatorname{diag}\left(\omega_{n i}^{2}, \quad i=1,2, \ldots, N\right)
\end{aligned}
$$

with damping $\zeta_{i}$ and natural frequency $\omega_{n i}$.

For simplicity, let

$$
\vartheta=\left[\begin{array}{l}
\eta \\
\psi
\end{array}\right]
$$

where $\psi=\dot{\eta}+\delta \omega$. The relative dynamic equations (11) can be written as [31]

$$
\begin{aligned}
& \dot{\omega}=J_{m b}^{-1}\left(-\omega^{\times} J_{m b} \omega-\delta^{T} K \delta \omega\right.
\end{aligned}
$$

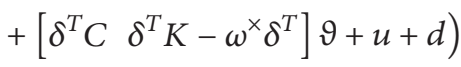

$$
\begin{aligned}
& \dot{\vartheta}=A \vartheta+B \omega \text {. }
\end{aligned}
$$

The matrices $J_{m b}, A$, and $B$ are given as

$$
\begin{aligned}
J_{m b} & =J-\delta^{T} \delta, \\
A & =\left[\begin{array}{cc}
0_{4 \times 4} & I_{4} \\
-C & -K
\end{array}\right], \\
B & =\left[\begin{array}{c}
-\delta \\
K \delta
\end{array}\right],
\end{aligned}
$$

where $0_{n \times m}$ is the $n \times m$ zero matrix and $I_{4}$ the $4 \times 4$ identity matrix. Clearly, $A$ is a Hurwitz matrix.

If we let

$$
\epsilon\left(q_{0}, q\right)=\left[\begin{array}{c}
1-\left|q_{0}\right| \\
q
\end{array}\right]
$$

then, the kinematic equation in terms of $\epsilon\left(q_{0}, q\right)$ can be expressed as

$$
\dot{\epsilon}\left(q_{0}, q\right)=\frac{1}{2} \Phi^{T}\left(q_{0}, q\right) \omega
$$

where

$$
\Phi\left(q_{0}, q\right)=\left[\begin{array}{c}
\operatorname{sign}\left(q_{0}\right) q^{T} \\
q^{\times}+q_{0} I_{3}
\end{array}\right]^{T}
$$

with

$$
\operatorname{sign}\left(q_{0}\right)= \begin{cases}1, & q_{0} \geq 0 \\ -1 & q_{0}<0\end{cases}
$$


Define a new variable $z\left(\omega, q_{0}, q\right)$ as

$$
z\left(\omega, q_{0}, q\right)=\omega+K_{1} \Phi\left(q_{0}, q\right) \epsilon\left(q_{0}, q\right)
$$

where $K_{1} \in \mathscr{R}^{3 \times 3}$ is a symmetric positive definite matrix. It should be noted that

$$
\begin{aligned}
\Phi & \left(q_{0}, q\right) \epsilon\left(q_{0}, q\right) \\
& =\left[\begin{array}{c}
\operatorname{sign}\left(q_{0}\right) q^{T} \\
q^{\times}+q_{0} I_{3}
\end{array}\right]^{T}\left[\begin{array}{c}
1-\operatorname{sign}\left(q_{0}\right) q_{0} \\
q
\end{array}\right] \\
& =\operatorname{sign}\left(q_{0}\right) q .
\end{aligned}
$$

Therefore, the variable $z\left(\omega, q_{0}, q\right)$ in $(21)$ becomes

$$
z\left(\omega, q_{0}, q\right)=\omega+\operatorname{sign}\left(q_{0}\right) K_{1} q
$$

The first time derivative of $z$ is obtained as

$$
\begin{aligned}
& \dot{z}\left(\omega, q_{0}, q\right)=J_{m b}^{-1}\left(-\omega^{\times} J_{m b} \omega-\delta^{T} K \delta \omega\right. \\
& +\frac{1}{2} \operatorname{sign}\left(q_{0}\right) J_{m b} K_{1} T(\bar{q}) \omega
\end{aligned}
$$

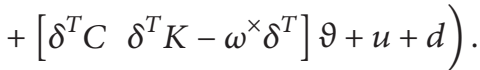

Consider the flexible spacecraft systems in (18), (15), and (24). We can rewrite these equations in the state-space form as

$$
\dot{x}=f(x)+g(x) u+g(x) d,
$$

where

$$
\begin{aligned}
x= & {\left[\begin{array}{l}
z \\
\epsilon \\
\vartheta
\end{array}\right], } \\
f(x)= & {\left[\begin{array}{c}
J_{m b}^{-1} \Xi\left(\omega, q_{0}, q\right) \\
\frac{1}{2} \Phi^{T}\left(q_{0}, q\right) \omega \\
A \vartheta+B \omega
\end{array}\right], } \\
b(x)= & {\left[\begin{array}{c}
J_{m b}^{-1} \\
0_{4 \times 3} \\
0_{8 \times 3}
\end{array}\right], } \\
\Xi\left(\omega, q_{0}, q\right)= & -\omega^{\times} J_{m b} \omega-\delta^{T} K \delta \omega \\
& +\frac{1}{2} \operatorname{sign}\left(q_{0}\right) J_{m b} K_{1} T(\bar{q}) \omega+H \vartheta
\end{aligned}
$$

with

$$
H=\left[\begin{array}{lll}
\delta^{T} C & \delta^{T} K-\omega^{\times} \delta^{T}
\end{array}\right] .
$$

3.3. Control Objective. In this work we consider rest-to-rest maneuvers of a flexible spacecraft. The control objective is to design a controller $u$ to achieve the desired rotations of flexible spacecraft in the presence of external disturbances and actuator saturation. In other words, we will find a controller $u$ to achieve

$$
\begin{aligned}
& \lim _{t \rightarrow \infty} q_{0}= \pm 1, \\
& \lim _{t \rightarrow \infty} q=0, \\
& \lim _{t \rightarrow \infty} \omega=0, \\
& \lim _{t \rightarrow \infty} \eta=0, \\
& \lim _{t \rightarrow \infty} \dot{\eta}=0
\end{aligned}
$$

that is, $q \rightarrow 0, q_{0} \rightarrow \pm 1, \omega \rightarrow 0, \eta \rightarrow 0$, and $\dot{\eta} \rightarrow 0$ as $t \rightarrow \infty$.

\section{Inverse Optimal Controller Design for Flexible Spacecraft}

In this section, we now propose an inverse optimal controller for stabilizing the complete attitude motion of flexible spacecraft in the presence of external disturbances.

In order to design a stabilizing controller for solving the inverse optimal problem with an input constraint we first choose a CLF for system (25) as the following candidate positive definite function:

$$
V(x)=\frac{\beta}{2} \epsilon^{T} \epsilon+\frac{1}{2} z^{T} J_{m b} z+\frac{1}{2} \vartheta^{T} P \vartheta,
$$

where $\beta$ is a positive constant and $P \in \mathscr{R}^{8 \times 8}$ is a positive definite matrix that is a solution of the Lyapunov equation $A^{T} P+P A=-Q$ with a positive definite matrix $Q \in \mathscr{R}^{8 \times 8}$.

The following assumptions are required in the subsequent controller design.

Assumption 4. The known matrices $J$ and $J_{m b}$ are symmetric positive definite and bounded.

Assumption 5. The control input torque of actuator $u \in \mathscr{R}^{3}$ satisfies $\|u\| \leq u_{\max }$, where $u_{\max }>0$ is a known constant.

It should be noted that, in practice, $u_{\max }$ is the maximum allowable torque input which actuators can produce.

We next show that the function $V(x)$ defined in (29) is a CLF for system (25) by using the following theorem.

Theorem 6. In the absence of disturbance vector $d$ in (25), under Assumptions 4 and 5, the positive definite function $V$ defined in (29) is a CLF for the spacecraft motion equation (25), if $\beta, K_{1}$, and $Q$ satisfy the following inequality:

$$
\Omega_{2}=\left[\begin{array}{cc}
\beta \lambda_{\min }\left(K_{1}\right) & -\frac{1}{2}\left\|P B K_{1}\right\| \\
-\frac{1}{2}\left\|P B K_{1}\right\| & \frac{1}{2} \lambda_{\min }(Q)
\end{array}\right]>0,
$$


where $\lambda_{\min }\left(K_{1}\right)$ and $\lambda_{\max }\left(K_{1}\right)$ denote the minimum and maximum singular values of the matrix $K_{1}$.

Proof. Since $J_{m b}$ is symmetric positive definite, $V(x)$ can be written as

$$
V=x^{T} \Omega_{1} x
$$

where

$$
\Omega_{1}=\frac{1}{2}\left[\begin{array}{ccc}
\beta I_{4} & 0_{4 \times 3} & 0_{4 \times 8} \\
0_{3 \times 4} & J_{m b} & 0_{3 \times 8} \\
0_{8 \times 4} & 0_{8 \times 3} & P
\end{array}\right] .
$$

With $\beta>0$ and $J_{m b}>0$, this ensures that $V(x)$ is positive definite. Taking the first derivative of $V$ with respect to time, we have

$$
\dot{V}=\beta \epsilon^{T} \dot{\epsilon}+\mathcal{\vartheta}^{T} P \dot{\vartheta}+z^{T} J_{m b} \dot{z},
$$

where by substituting (18), (15), and (24) into (33) one obtains

$$
\begin{aligned}
\dot{V} & =\beta \epsilon^{T} \Phi^{T}\left(z-K_{1} \Phi \epsilon\right)+\vartheta^{T} P(A \vartheta+B \omega) \\
& +z^{T}\left[-\omega^{\times} J_{m b} \omega-\delta^{T} K \delta \omega\right. \\
& +\frac{1}{2} \operatorname{sign}\left(q_{0}\right) J_{m b} K_{1} T(\bar{q}) \omega \\
& \left.+\left[\delta^{T} C \quad \delta^{T} K-\omega^{\times} \delta^{T}\right] \vartheta\right]+z^{T} u=-\beta \epsilon^{T} \Phi^{T} K_{1} \Phi \epsilon \\
& -\frac{1}{2} \vartheta^{T} Q \vartheta-z^{T}\left(\frac{1}{2} \operatorname{sign}\left(q_{0}\right) J_{m b} K_{1} T(\bar{q}) K_{1}\right. \\
& \left.-\delta^{T} K \delta K_{1}\right)(\Phi \epsilon)-z^{T} \omega^{\times} J_{m b} z-\beta z^{T} \Phi \epsilon \\
& +z^{T} \omega^{\times} J_{m b} K_{1} \Phi \epsilon+z^{T}\left[\delta^{T} C \delta^{T} K+\left(K_{1} \Phi \epsilon\right) \delta^{T}\right] \vartheta \\
& +z^{T}\left[0-z^{\times} \delta^{T}\right] \vartheta+z^{T} H \vartheta+\vartheta^{T} P B z-\vartheta^{T} P B K_{1} \Phi \epsilon \\
& -z^{T} \delta^{T} K \delta z+z^{T} \frac{1}{2} \operatorname{sign}\left(q_{0}\right) J_{m b} K_{1} T(\bar{q}) z .
\end{aligned}
$$

Using the fact that $z^{T}\left[\begin{array}{ll}0 & -z^{\times} \delta^{T}\end{array}\right] \vartheta=0,\|T(\bar{q})\|=1$ and $\|\Phi\|=$ 1 , we obtain

$$
\begin{aligned}
\dot{V} & \leq-\beta \lambda_{\min }\left(K_{1}\right)\|\Phi \epsilon\|^{2}+\left(\left\|\frac{1}{2} J_{m b} K_{1}\right\|+\left\|\delta^{T} K \delta\right\|\right. \\
& \left.+\left\|\omega^{\times} J_{m b}\right\|\right)\|z\|^{2}-\frac{1}{2} \lambda_{\min }(Q)\|\vartheta\|^{2} \\
& +\left(\left\|\frac{1}{2} J_{m b} K_{1}\right\|\left\|K_{1}\right\|+\left\|\delta^{T} K \delta\right\|\left\|K_{1}\right\|\right. \\
& \left.+\left\|\omega^{\times} J_{m b}\right\|\left\|K_{1}\right\|+\beta\right)\|z\|\|\Phi \epsilon\|-\left\|\omega^{\times} J_{m b}\right\|\|z\|^{2} \\
& +\left(\left\|\left[\delta^{T} C \quad \delta^{T} K\right]\right\|+\|P B\|\right)\|z\|\|\vartheta\|+\left\|P B K_{1}\right\|\|\vartheta\| \\
& \cdot\|\Phi \epsilon\|+z^{T} u .
\end{aligned}
$$

We know that the first time derivative of $V(x)$ can be expressed as

$$
\dot{V}=L_{f} V+L_{g} V u \text {. }
$$

Comparing (35) and (36), it follows that

$$
L_{g} V=z^{T} .
$$

Therefore, if $L_{g} V=0$, then we have

$$
\begin{aligned}
z & =0_{6 \times 1}, \\
\|z\| & =0 .
\end{aligned}
$$

Next, we show that if $L_{g} V=0$, then $L_{f} V<0$ for all $x \neq 0$. Substituting $z=0_{6 \times 1}$ in (34), $L_{f} V$ can be expressed as

$$
\begin{aligned}
L_{f} V= & \dot{V} \\
\leq & -\beta \lambda_{\min }\left(K_{1}\right)\|\Phi \epsilon\|^{2}-\frac{1}{2} \lambda_{\min }(Q)\|\vartheta\|^{2} \\
& +\|\vartheta\|\left\|P B K_{1}\right\|\|\Phi \epsilon\| \\
= & -\beta \lambda_{\min }\left(K_{1}\right)\|\varrho\|^{2}-\frac{1}{2} \lambda_{\min }(Q)\|\vartheta\|^{2} \\
& +\|\vartheta\|\left\|P B K_{1}\right\|\|\varrho\|=-\omega^{T} \Omega_{2} \omega,
\end{aligned}
$$

where $\varrho=\Phi \epsilon$ and $\Phi=[\|\varrho\|\|\vartheta\|]^{T}$. It follows that if condition (30) is satisfied, then $L_{f} V<0, \forall x \neq 0$ is achieved. This guarantees that the candidate $V(x)$ is a CLF for the spacecraft motion system (25). This completes the proof.

Next, the main results of our proposed inverse optimal control for the spacecraft model are presented. The presented dynamic feedback control law is designed as

$$
\begin{aligned}
u & =-R^{-1}\left(\omega, q_{0}, q\right) z \\
& = \begin{cases}-\frac{u_{\max }}{2}\|z\| \operatorname{sign}(z) & \text { if }\|u\|>u_{\max } \\
-\frac{\tau}{2} & \text { if }\|u\| \leq u_{\max }\end{cases}
\end{aligned}
$$

where

$$
\tau= \begin{cases}\frac{L_{f} V(x)+\sqrt{\left(L_{f} V(x)\right)^{2}+\left(z^{T} z\right)^{2}}}{\|z\|^{2}}, & \|z\| \neq 0 \\ 0, & \|z\|=0\end{cases}
$$

with

$$
\begin{aligned}
L_{f} V(x)= & \beta \epsilon^{T} \Phi^{T}\left(z-K_{1} \Phi \epsilon\right)+\vartheta^{T} P A \vartheta \\
& +\vartheta^{T} P B\left(z-K_{1} \Phi \epsilon\right)+z^{T} \Xi, \\
\operatorname{sign}(z)= & {\left[\operatorname{sign}\left(z_{1}\right) \operatorname{sign}\left(z_{2}\right) \operatorname{sign}\left(z_{3}\right)\right]^{T} . }
\end{aligned}
$$

It should be noted that the Lyapunov stability theory needs the existence and uniqueness of a solution, for an 
initial condition. This is an important basis for the subsequent analysis of this paper. The following theorem shows that the system consisting of (15), (18), and (24) satisfies existence and uniqueness solution requirement since the system is locally Lipschitz [32].

Theorem 7. In the absence of disturbance, the spacecraft attitude system consisting of (15), (18), and (24) is locally Lipschitz in $(\epsilon, z, 9) \in \mathscr{R}^{4} \times \mathscr{R}^{3} \times \mathscr{R}^{8}$ and satisfies existence and uniqueness solution requirement.

Proof. We know that $\|z\|=\left\|\omega+K_{1} q\right\| \geq\|\omega\|$ and $\|T(\bar{q})\|=1$ and these properties will be used in this proof. Since (15) and (18) are continuously differentiable, the following inequalities can be obtained:

$$
\begin{aligned}
& \left\|\dot{\vartheta}_{1}-\dot{\vartheta}_{2}\right\| \leq l_{1}\left\|\left[\begin{array}{c}
\omega_{1}-\omega_{2} \\
\vartheta_{1}-\vartheta_{2}
\end{array}\right]\right\| \leq l_{1}\left\|\left[\begin{array}{c}
\epsilon_{1}-\epsilon_{2} \\
z_{1}-z_{2} \\
\vartheta_{1}-\vartheta_{2}
\end{array}\right]\right\|, \\
& \left\|\dot{\epsilon}_{1}-\dot{\epsilon}_{2}\right\| \leq l_{2}\left\|\left[\begin{array}{c}
\epsilon_{1}-\epsilon_{2} \\
\omega_{1}-\omega_{2}
\end{array}\right]\right\| \leq l_{2}\left\|\left[\begin{array}{c}
\epsilon_{1}-\epsilon_{2} \\
z_{1}-z_{2} \\
\vartheta_{1}-\vartheta_{2}
\end{array}\right]\right\|,
\end{aligned}
$$

where $l_{1}, l_{2} \in \mathscr{R}^{+}$. It should be noted that in (19) the term $\operatorname{sign}\left(q_{0}\right)$ only provides the negative or positive value of $q$ and it has no effect on the differentiation. Thus, the term $\operatorname{sign}\left(q_{0}\right) q^{T}$ is continuously differentiable.

Now, we consider system (24) with control input $u=R^{-1} z$ and obtain

$$
\begin{aligned}
\left\|J_{m b} \dot{z}_{1}-J_{m b} \dot{z}_{2}\right\| \leq & \left\|-\omega_{1}^{\times} J_{m b} \omega_{1}+\omega_{2}^{\times} J_{m b} \omega_{2}\right\| \\
& +\lambda_{\max }\left(\delta^{T} K \delta\right)\left\|\omega_{1}-\omega_{2}\right\| \\
& +\lambda_{\max }\left(\frac{1}{2} J_{m b} K_{1}\right)\left\|\omega_{1}-\omega_{2}\right\| \\
& +\lambda_{\max }(H)\left\|\vartheta_{1}-\vartheta_{2}\right\| \\
& +\lambda_{\max }\left(R^{-1}\right)\left\|z_{1}-z_{2}\right\| .
\end{aligned}
$$

Since the terms $-\omega_{1}^{\times} J_{m b} \omega_{1}$ and $-\omega_{2}^{\times} J_{m b} \omega_{2}$ are continuously differentiable, one obtains

$$
\begin{aligned}
& \left\|-\omega_{1}^{\times} J_{m b} \omega_{1}+\omega_{2}^{\times} J_{m b} \omega_{2}\right\| \leq l_{3}\left\|\omega_{1}-\omega_{2}\right\| \\
& \leq l_{3}\left\|\left[\begin{array}{c}
\epsilon_{1}-\epsilon_{2} \\
z_{1}-z_{2} \\
\vartheta_{1}-\vartheta_{2}
\end{array}\right]\right\|,
\end{aligned}
$$

Now, $\left\|J_{m b} \dot{z}_{1}-J_{m b} \dot{z}_{2}\right\|$ can be rearranged as

$$
\left\|J_{m b} \dot{z}_{1}-J_{m b} \dot{z}_{2}\right\| \leq L_{1}\left\|\left[\begin{array}{c}
\epsilon_{1}-\epsilon_{2} \\
z_{1}-z_{2} \\
\vartheta_{1}-\vartheta_{2}
\end{array}\right]\right\|,
$$

where $L_{1}=l_{3}+\lambda_{\max }\left(\delta^{T} K \delta\right)+(1 / 2) \lambda_{\max }\left(J_{m b} K_{1}\right)+\lambda_{\max }(H)+$ $\lambda_{\text {max }}\left(R^{-1}\right)$.

Recalling (43), the following inequalities can be obtained:

$$
\left\|\left[\begin{array}{c}
\dot{\epsilon}_{1}-\dot{\epsilon}_{2} \\
J_{m b} \dot{z}_{1}-J_{m b} \dot{z}_{2} \\
\dot{\vartheta}_{1}-\dot{\vartheta}_{2}
\end{array}\right]\right\|^{2} \leq\left(l_{1}^{2}+l_{2}^{2}+L_{1}^{2}\right)\left\|\left[\begin{array}{c}
\epsilon_{1}-\epsilon_{2} \\
z_{1}-z_{2} \\
\vartheta_{1}-\vartheta_{2}
\end{array}\right]\right\|^{2}
$$

which is equivalent to

$$
\left\|\left[\begin{array}{c}
\dot{\epsilon}_{1}-\dot{\epsilon}_{2} \\
J_{m b} \dot{z}_{1}-J_{m b} \dot{z}_{2} \\
\dot{\vartheta}_{1}-\dot{\vartheta}_{2}
\end{array}\right]\right\| \leq L_{2}\left\|\left[\begin{array}{c}
\epsilon_{1}-\epsilon_{2} \\
z_{1}-z_{2} \\
\vartheta_{1}-\vartheta_{2}
\end{array}\right]\right\|,
$$

where $L_{2}=\sqrt{l_{1}^{2}+l_{2}^{2}+L_{1}^{2}}$. The last inequality (48) states that the spacecraft attitude system consisting of (15), (18), and (24) is locally Lipschitz in $(\epsilon, z, \vartheta) \in \mathscr{R}^{4} \times \mathscr{R}^{3} \times \mathscr{R}^{8}$. Hence, the existence of a unique solution is guaranteed.

Remark 8. In this paper, the disturbance $d$ in (24) will be estimated by the proposed observer in Section 5. With the estimation result, the disturbance $d$ will be canceled out by the estimated one. Thus, it makes sense to ignore the disturbance $d$ in Theorem 7 and subsequent analysis.

Theorem 9. The control law (40) stabilizes the spacecraft system (25) if the parameters $\beta, K_{1}, u_{\max }$, and $Q$ satisfy the following inequality:

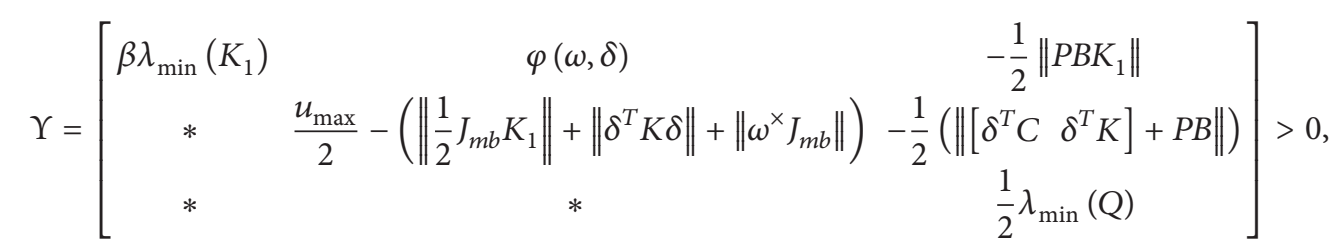

$$
\begin{aligned}
& u_{\max }>\left\|J_{m b} K_{1}\right\|+2\left\|\delta^{T} K \delta\right\|+2\left\|\omega^{\times} J_{m b}\right\|,
\end{aligned}
$$


where $\varphi(\omega, \delta)=-(1 / 2)\left((1 / 2)\left\|J_{m b} K_{1}\right\|\left\|K_{1}\right\|+\left\|\omega^{\times} J_{m b}\right\|\left\|K_{1}\right\|+\right.$ $\left.\left\|\delta^{T} K \delta\right\|\left\|K_{1}\right\|+\beta\right)$.

Proof. It is required to show that the control law $u$ in (40) is a stabilizing controller for attitude control system (25). Consider the smooth positive definite radially unbounded Lyapunov function as (29).

For the case $\|u\| \leq u_{\max }$, the time derivative of a CLF $V(x)$ is

$$
\begin{aligned}
\dot{V} & =\left[\frac{\partial V}{\partial x}\right]^{T}(f(x)+g(x) u) \\
& \leq L_{f} V-\frac{1}{2} z^{T} \frac{L_{f} V(x)+\sqrt{\left(L_{f} V(x)\right)^{2}+\left(z^{T} z\right)^{2}}}{\|z\|^{2}} z \\
& =L_{f} V-\frac{1}{2}\left(L_{f} V(x)+\sqrt{\left(L_{f} V(x)\right)^{2}+\left(z^{T} z\right)^{2}}\right) \\
& =\frac{1}{2}\left(L_{f} V-\sqrt{\left(L_{f} V(x)\right)^{2}+\left(z^{T} z\right)^{2}}\right) .
\end{aligned}
$$

For the definition of CLF, for $z=0$ we obtain $\dot{V}=L_{f} V(x)<0$ for all $x \neq 0$. For $z \neq 0, \dot{V}<0$ is achieved for all $x \neq 0$ because $L_{f} V<\sqrt{\left(L_{f} V(x)\right)^{2}+\left(z^{T} z\right)^{2}}$ is always satisfied. Therefore, $\dot{V}<0$ is achieved for all $x \neq 0$.

For the case $\|u\|>u_{\max }$, substituting $u=u_{\max }$ into (35), the time derivative of a CLF $V(x)$ becomes

$$
\begin{aligned}
\dot{V} & \leq-\beta \lambda_{\min }\left(K_{1}\right)\|\varrho\|^{2}+\left(\left\|\frac{1}{2} J_{m b} K_{1}\right\|+\left\|\delta^{T} K \delta\right\|\right)\|z\|^{2} \\
& -\frac{1}{2} \lambda_{\min }(Q)+\left\|P B K_{1}\right\|\|z\|\|\vartheta\|+\left(\left\|\frac{1}{2} J_{m b} K_{1}\right\|\left\|K_{1}\right\|\right. \\
& \left.+\left\|\delta^{T} K \delta\right\|\left\|K_{1}\right\|+\left\|\omega^{\times} J_{m b}\right\|\left\|K_{1}\right\|+\beta\right)\|z\|\|\varrho\| \\
& +\left\|\omega^{\times} J_{m b}\right\|\|z\|^{2}+\left(\left\|\left[\delta^{T} C \delta^{T} K\right]\right\|+\|P B\|\right)\|\vartheta\|\|\varrho\| \\
& -\frac{1}{2} u_{\max } z^{T} z \leq-\lambda_{\min }\left(K_{1}\right)\|\varrho\|^{2}-\left(\frac{u_{\max }}{2}\right. \\
& \left.-\left(\left\|\frac{1}{2} J_{m b} K_{1}\right\|+\left\|\delta^{T} K \delta\right\|+\left\|\omega^{\times} J_{m b}\right\|\right)\right)\|z\|^{2} \\
& -\lambda_{\min }(Q) \frac{\|\vartheta\|^{2}}{2}+\left(\left\|\frac{1}{2} J_{m b} K_{1}\right\|\left\|K_{1}\right\|\right. \\
& \left.+\left\|\delta^{T} K \delta\right\|\left\|K_{1}\right\|+\left\|\omega^{\times} J_{m b}\right\|\left\|K_{1}\right\|+\beta\right)\|z\|\|\varrho\| \\
& +\left(\left\|\left[\delta^{T} C \delta^{T} K\right]\right\|+\|P B\|\right)\|z\|\|\vartheta\|+\left\|P B K_{1}\right\|\|\vartheta\| \\
& +\|\varrho\|,
\end{aligned}
$$

which can be further written as

$$
\dot{V} \leq-\zeta \Upsilon \zeta
$$

where $\zeta=[\|\varrho\|\|z\|\|9\|]^{T}$. Evidently, if (49) and (50) are satisfied, one obtains $\dot{V}<0$ for all $x \neq 0$. Therefore, controller (40) asymptotically stabilizes the spacecraft system (25).

Remark 10. Inequality (30) is considered as the special case of inequality (49). When $z=0$ is set, inequality (49) is reduced to a more simple form as presented in (30). Thus, in the controller design, it is sufficient to select the parameters $\beta, K_{1}, u_{\max }$, and $Q$ such that the inequalities (49) and (50) are satisfied. This automatically satisfies condition (30).

Remark 11. When the disturbance vector $d$ is ignored, the proposed control law (40) can stabilize the two equilibrium points $\bar{q}=\left[\begin{array}{llll}1 & 0 & 0 & 0\end{array}\right]^{T}$ and $\bar{q}=\left[\begin{array}{llll}-1 & 0 & 0 & 0\end{array}\right]^{T}$. If the initial $q_{0}(0)<0$ is chosen, the proposed law will force $q_{0}$ to -1 . On the other hand, if the initial $q_{0}(0)>0$ is selected, the proposed law will drive $q_{0}$ to 1 . This means that the proposed law can avoid an unwinding phenomenon. However, most existing inverse optimal control methods (see, e.g., $[13,22,33]$ ) could only stabilize one equilibrium point $\bar{q}=\left[\begin{array}{llll}1 & 0 & 0 & 0\end{array}\right]^{T}$ and another equilibrium point $\bar{q}=\left[\begin{array}{llll}-1 & 0 & 0 & 0\end{array}\right]^{T}$ is unstable. If the initial $q_{0}(0)<0$ is chosen, these controllers would force $q_{0}$ to 1 instead of -1 , so an unwinding phenomenon may occur.

Next, when $d$ in (25) is ignored, it can be shown that the feedback stabilizing controller $u$ defined in (40) solves the inverse optimal control problem.

Theorem 12. The following dynamic feedback control law

$$
\begin{aligned}
u & =\kappa^{*}(x)=-2 R^{-1}\left(\omega, q_{0}, q\right) z \\
& = \begin{cases}-u_{\max }\|z\| \operatorname{sign}(z) & \text { if }\|u\|>u_{\max } \\
-\tau & \text { if }\|u\| \leq u_{\max }\end{cases}
\end{aligned}
$$

solves the inverse optimal assignment problem for the attitude control system equation (25) by minimizing the cost functional

$$
L_{a}=\lim _{T \rightarrow \infty}\left[4 V(T)+\int_{0}^{T}\left(l(x)+u^{T} R u\right) d t\right],
$$

where $l(x)$ is defined by

$$
\begin{aligned}
& l(x)=-4\left[\beta \epsilon^{T} \Phi^{T}\left(z-K_{1} \Phi \epsilon\right)+\vartheta^{T} P A \vartheta\right. \\
& \left.+\vartheta^{T} P B\left(z-K_{1} \Phi \epsilon\right)+z^{T} \Xi\right]+4 z^{T} R^{-1} z .
\end{aligned}
$$

and $T$ is a final time.

In practice, the time instant $T$ in (55) is the time period of a complete rotation of the spacecraft. Also, the proof of Theorem 12 is given.

Proof. With the derivations in Theorem 9 and the definitions of $R(x)$ and $l(x)$, we obtain that $R(x)$ is positive definite and

$$
l(x)=-4 \dot{V}
$$

which shows that $l(x)$ is positive semidefinite. Although we have two cases, $\|u\|>u_{\max }$ and $\|u\| \leq u_{\max }$, both cases obtain 
$\dot{V}<0$, for all $x \neq 0$. This means $l(x)$ is positive semidefinite for $\|u\|>u_{\max }$ and $\|u\| \leq u_{\max }$. Therefore, $L_{a}$ is a meaningful cost functional for the attitude control problem of flexible spacecraft, penalizing on $x$ as well as the control effort $u$. Hence, the control input (54) minimizes the cost functional $L_{a}$. One has the optimal cost $L_{a}^{*}=4 V(0)$ which is obtained by substituting $l(x)$ into (55). This completes the proof.

\section{Robust Inverse Optimal Control with Sliding Mode Disturbance Observer}

Recently, because of the great advances in nonlinear control theory, the extended state observer (ESO) $[32,34]$ has high efficiency in accomplishing nonlinear dynamic estimation. The main idea of ESO is the total disturbance vector representing system uncertainties and disturbances are considered as an added state of the system; then all states of the system including the added one will be observed accurately and rapidly. However, few rigorous proofs of ESO convergence have been studied. In this section a sliding mode disturbance observer (SMDO) which uses similar structure to the traditional ESO is presented and the ultimate boundedness of the proposed disturbance observer is ensured using the Lyapunov technique.

5.1. Sliding Mode Disturbance Observer. We now consider the following plant system as

$$
\begin{gathered}
\dot{Z}_{1}=Z_{2} \\
\dot{Z}_{2}=Z_{3} \\
\vdots \\
\dot{Z}_{n}=\xi(t)+F(Z)+G(Z) u \\
y=Z_{1},
\end{gathered}
$$

where $Z=\left[\begin{array}{llll}Z_{1} & Z_{2} & \cdots & Z_{n}\end{array}\right]^{T} \in \mathscr{R}^{n}, u \in \mathscr{R}$, and $y \in \mathscr{R}$ are the states, input, and output of the system, respectively. $\xi(t)$ is the unknown total disturbance including disturbances and unknown part of the system, $F(Z)$ denotes the known part of the plant model. We add an extended state $Z_{n+1}$ as the total disturbance $\xi(t)$ to system (58). The new SMDO for system (58) is constructed as

$$
\begin{gathered}
E=y-\widehat{Z}_{1} \\
\dot{\bar{Z}}_{1}=\widehat{Z}_{2}+\lambda_{1} \operatorname{sign}(E) \\
\dot{\bar{Z}}_{2}=\widehat{Z}_{3}+\lambda_{2} \operatorname{sign}(E) \\
\vdots \\
\dot{\bar{Z}}_{n}=\xi(t)+F(Z)+G(Z) u+\lambda_{n} \operatorname{sign}(E) \\
\dot{\vec{Z}}_{n+1}=h(t)+\lambda_{n+1} \operatorname{sign}(E),
\end{gathered}
$$

where $\widehat{Z}=\left[\begin{array}{llll}\widehat{Z}_{1} & \widehat{Z}_{2} & \cdots & \widehat{Z}_{n+1}\end{array}\right]^{T} \in \mathscr{R}^{n+1}$ and $\lambda_{i}, i=$ $1,2, \ldots, n+1$, are the observer gain parameters to be selected. $\widehat{Z}_{n+1}$ is the observer variable used to estimate the total disturbance, $h(t)$ denotes the first time derivative of the total disturbance $\xi(\widehat{Z}, t)$, and $E$ is the estimation error of the SMDO. The following assumption must be included to ensure the convergence of the proposed SMDO.

Assumption 13. The total disturbance $\xi(t)$ in (58) and its first time derivative $h(t)$ are unknown but bounded; that is, $\|\xi(t)\| \leq \bar{\xi}$ and $\|h(t)\| \leq \bar{h}$, where $\bar{\xi}$ and $\bar{h}$ denote positive constants.

The convergence of the states of proposed SMDO to the states of plant (58) is investigated in the following theorem.

Theorem 14. Let Assumptions 4, 5, and 13 hold. Consider system (58) with the SMDO (59). Then, there exist positive observer gains $\lambda_{1}, \lambda_{2}, \ldots, \lambda_{n+1}$ such that the ultimate boundedness of the observed errors is ensured.

Proof. To investigate the stability of the SMDO system, one must consider an expression for the observer error dynamics. We first define the observer errors $\widetilde{Z}_{1}=Z_{1}-\widehat{Z}_{1}, \widetilde{Z}_{2}=Z_{2}$ $\widehat{Z}_{2}, \ldots, \widetilde{Z}_{n}=Z_{2}-\widehat{Z}_{n}$, and $\widetilde{Z}_{n+1}=Z_{n+1}-\xi(t)$. The observer error dynamics can be expressed as

$$
\begin{gathered}
\dot{\bar{Z}}_{1}=\widetilde{Z}_{2}-\lambda_{1} \operatorname{sign}(E) \\
\dot{\bar{Z}}_{2}=\widetilde{Z}_{3}-\lambda_{2} \operatorname{sign}(E) \\
\vdots \\
\dot{\bar{Z}}_{n}=\widetilde{Z}_{n+1}-\lambda_{n} \operatorname{sign}(E) \\
\dot{\bar{Z}}_{n+1}=-h(t)-\lambda_{n+1} \operatorname{sign}(E) .
\end{gathered}
$$

We now show that the ultimate boundedness of observed states $\widetilde{Z}_{i}, i=1,2, \ldots, n+1$, is guaranteed. The sliding surface is defined as $S\left(\widetilde{Z}_{1}\right)=\widetilde{Z}_{1}=0$. Consider the following Lyapunov function:

$$
\mathscr{V}_{1}=\frac{1}{2} S^{2} .
$$

The first time derivative of $\mathscr{V}_{1}$ is

$$
\begin{aligned}
\dot{\mathscr{V}}_{1} & =\widetilde{Z}_{1} \dot{\bar{Z}}_{1} \leq \widetilde{Z}_{1}\left(\widetilde{Z}_{2}-\lambda_{1} \operatorname{sign}(E)\right) \\
& \leq\left|\widetilde{Z}_{2}\right|\left|\widetilde{Z}_{1}\right|-\lambda_{1}\left|\widetilde{Z}_{1}\right|=-\left|\widetilde{Z}_{1}\right|\left(\lambda_{1}-\left|\widetilde{Z}_{2}\right|\right) .
\end{aligned}
$$

We could select $\lambda_{1}>\left|\widetilde{Z}_{2}\right|+\varepsilon_{1}$, where $\varepsilon_{1}>0$. Then, $\dot{\mathscr{V}}_{1} \leq$ $-\left|\widetilde{Z}_{1}\right| \varepsilon_{1}$. This would guarantee that $\dot{\mathscr{V}} \leq 0, \forall \widetilde{Z}_{1} \neq 0$, and the finite time convergence of $\widetilde{Z}_{1}$ to the sliding surface $S=0$ is also ensured. When the sliding mode occurs (i.e., $\widetilde{Z}_{1}=0$ 
and $\dot{\bar{Z}}_{1}=0$ ), the resulting error system on the sliding mode becomes

$$
\begin{gathered}
\dot{\bar{Z}}_{2}=-\frac{\lambda_{2}}{\lambda_{1}} \widetilde{Z}_{2}+\widetilde{Z}_{3} \\
\dot{\bar{Z}}_{3}=-\frac{\lambda_{3}}{\lambda_{1}} \widetilde{Z}_{2}+\widetilde{Z}_{4} \\
\vdots \\
\dot{\vec{Z}}_{n}=-\frac{\lambda_{n}}{\lambda_{1}} \widetilde{Z}_{2}+\widetilde{Z}_{n+1} \\
\dot{\vec{Z}}_{n+1}=-\frac{\lambda_{n+1}}{\lambda_{1}} \widetilde{Z}_{2}-h(t) .
\end{gathered}
$$

Letting $\alpha_{i}=\lambda_{i} / \lambda_{1}$, then (63) can be written as

$$
\dot{X}=A_{1} X+B_{1} h,
$$

where $X=\left[\begin{array}{llll}\widetilde{Z}_{2} & \widetilde{Z}_{3} & \cdots & \widehat{Z}_{n+1}\end{array}\right]^{T} \in \mathscr{R}^{n}$,

$$
\begin{aligned}
A_{1} & =\left[\begin{array}{ccccc}
-\alpha_{2} & 1 & 0 & \ldots, 0 \\
-\alpha_{3} & 0 & 1 & \ldots, 0 \\
\vdots & \vdots & \ddots & \vdots & \vdots \\
-\alpha_{n} & 0 & 0 & \ldots, 1 \\
-\alpha_{n+1} & 0 & 0 & \ldots, 0
\end{array}\right], \\
B_{1} & =\left[\begin{array}{c}
0 \\
0 \\
\vdots \\
0 \\
-1
\end{array}\right] .
\end{aligned}
$$

If the matrix $A_{1}$ is Hurwitz, there exists a unique positive definite matrix $P_{1}$ such that $A_{1}^{T} P_{1}+P_{1} A_{1}=-Q_{1}$, where $Q_{1}$ is a positive definite matrix. Select the Lyapunov function as

$$
\mathscr{V}_{2}(X)=X^{T} P_{1} X
$$

Its first time derivative is

$$
\begin{aligned}
\dot{\mathscr{V}}_{2} & =2 X^{T} P_{1} \dot{X}=2 X^{T} P_{1}\left(A_{1} X+B_{1} h\right) \\
& =-X^{T} Q_{1} X+2 X^{T} P_{1} B_{1} h \\
& \leq-\lambda_{\text {min }}\left(Q_{1}\right)\|X\|^{2}+2 \bar{h} \lambda_{\text {max }}\left(P_{1}\right)\left\|B_{1}\right\|\|X\| .
\end{aligned}
$$

Using (66) we know that $\lambda_{\text {min }}\left(P_{1}\right)\|X\|^{2} \leq \mathscr{V}_{2} \leq$ $\lambda_{\max }\left(P_{1}\right)\|X\|^{2}$ and it follows that

$$
\begin{aligned}
\dot{\mathscr{V}}_{2} \leq & -\frac{\lambda_{\min }\left(Q_{1}\right)}{\lambda_{\max }\left(P_{1}\right)} \mathscr{V}_{2} \\
& +2 \bar{h} \lambda_{\max }\left(P_{1}\right)\left\|B_{1}\right\| \frac{\mathscr{V}_{2}^{1 / 2}}{\sqrt{\lambda_{\min }\left(P_{1}\right)}} .
\end{aligned}
$$

Setting $\mathscr{W}=\mathscr{V}_{2}^{2}$, one obtains

$$
\dot{\mathscr{W}} \leq-c_{1} \mathscr{W}+c_{2}
$$

where $c_{1}=\lambda_{\text {min }}\left(Q_{1}\right) / \lambda_{\text {max }}\left(P_{1}\right)$ and $c_{2}=2\left(\lambda_{\text {max }}\left(P_{1}\right)\left\|B_{1}\right\| \bar{h} /\right.$ $\left.\sqrt{\lambda_{\min }\left(P_{1}\right)}\right)$. This ensures that the observed errors $\widetilde{Z}_{i}, i=$ $1,2, \ldots, n+1$, converge to a small bound of zero.

5.2. Robust Inverse Optimal Control Design. We now consider system (24) and define the auxiliary dynamics

$$
Z_{1}=F\left(Z_{1}\right)+G\left(Z_{1}\right) u+\xi(t)
$$

where $Z_{1}=z, F\left(Z_{1}\right)=J_{m b}^{-1} \Xi, G\left(Z_{1}\right)=J_{m b}^{-1}$, and $\xi(t)=J_{m b}^{-1} d$. Applying the SMDO (59) with $n=1$, we have

$$
\begin{aligned}
& \dot{\widehat{Z}}_{1}=F\left(Z_{1}\right)+G\left(Z_{1}\right) u+\widehat{Z}_{2}+\lambda_{1} \operatorname{sign}(E) \\
& \dot{\vec{Z}}_{2}=h(t)+\lambda_{2} \operatorname{sign}(E) .
\end{aligned}
$$

Using the results from the SMDO, the estimated disturbance is determined by $\widehat{\xi}=G^{-1} \widehat{Z}_{2}$. Thus, the proposed robust inverse optimal control with an input constraint can be obtained as

$$
u=\kappa^{*}(x)-G^{-1} \widehat{Z}_{2} .
$$

With suitable control gains defined by the inverse optimal control approach based on CLF concept, the optimal feedback controller (72) contains both optimality and robustness performance to attenuate external disturbances.

\section{Simulation Results}

The rest-to-rest maneuver of flexible spacecraft is considered with numerical simulations to compare the performance of the proposed inverse optimal attitude control (proposed IOAC) and minimax inverse optimal attitude control (minimax IOAC) in [21]. The spacecraft is assumed to have the nominal inertia matrix [28]

$$
J=\left[\begin{array}{ccc}
350 & 3 & 4 \\
3 & 270 & 10 \\
4 & 10 & 190
\end{array}\right] \mathrm{kg} \cdot \mathrm{m}^{2}
$$

and coupling matrices

$$
\delta=\left[\begin{array}{ccc}
6.45637 & 1.27814 & 2.15629 \\
-1.25619 & 0.91756 & -1.67264 \\
1.11678 & 2.48901 & -0.83674 \\
1.23637 & -2.6581 & -1.12503
\end{array}\right] \mathrm{kg}^{1 / 2} \cdot \mathrm{m} / \mathrm{s}^{2}
$$




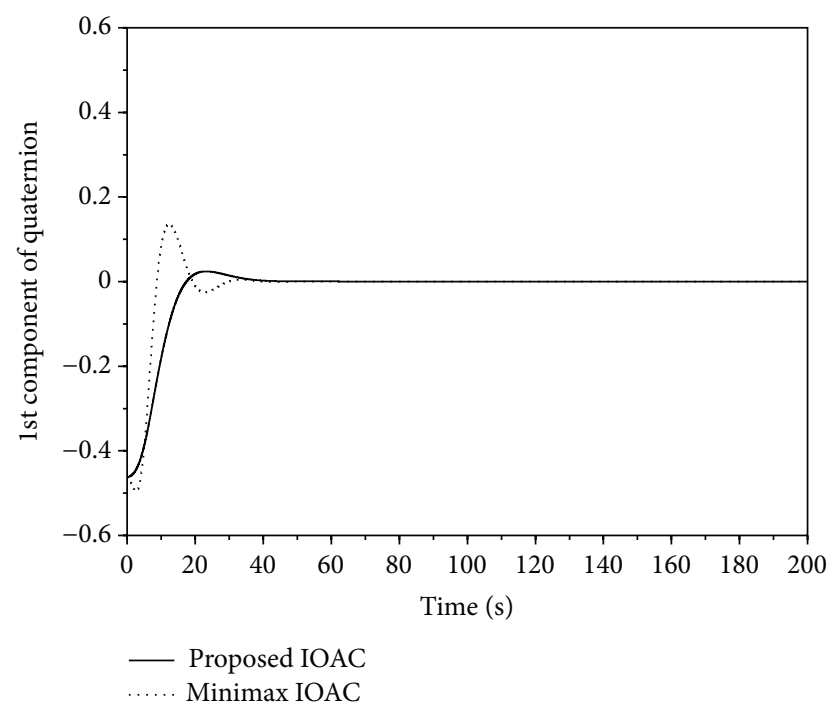

FIGURE 1: Time responses of the 1st component of quaternion.

respectively. The natural frequencies and damping ration are provided by

$$
\begin{aligned}
\omega_{n 1} & =0.7681, \\
\omega_{n 2} & =1.1038, \\
\omega_{n 3} & =1.8733, \\
\omega_{n 4} & =2.5496 \mathrm{rad} / \mathrm{sec} \\
\xi_{1} & =0.0056, \\
\xi_{2} & =0.0086, \\
\xi_{3} & =0.013, \\
\xi_{1} & =0.025 .
\end{aligned}
$$

The maximum torque input is restricted by $u_{\max }=5 \mathrm{~N}$ $\mathrm{m}$. The control parameters of the proposed controller (72) are set as $K_{1}=0.4 I_{3}, Q=5 I_{8}$, and $\beta=20$. Only the first four elastic modes have been considered in the controller design. The initial states of the rotation motion are given by $[21] \bar{q}(0)=\left[\begin{array}{llll}-0.3320 & -0.4618 & 0.1915 & 0.7999\end{array}\right]^{T}, \omega(0)=$ $\left[\begin{array}{lll}0 & 0 & 0\end{array}\right]^{T} \mathrm{rad} / \mathrm{sec}$, and $\vartheta(0)=\left[\begin{array}{llllllll}0 & 0 & 0 & 0 & 0 & 0 & 0 & 0\end{array}\right]^{T}$. For the minimax IOAC in [21], the control parameters $k_{q}=30$ and $k_{\omega}=18$ are chosen. The periodic disturbances are provided by

$$
d(t)=\left[\begin{array}{c}
0.3 \cos (0.1 t)+0.1 \\
0.15 \sin (0.1 t)+0.3 \cos (0.1 t) \\
0.3 \sin (0.1 t)+0.1
\end{array}\right] \mathrm{N}-\mathrm{m}
$$

Simulation results of the proposed IOAC and minimax IOAC in [21] are presented in Figures 1-14. From Figures 1-3 we can see that the proposed controller (72) offers smoother attitude responses. For the simulation, since the initial condition of $q_{0}$ is set as $q(0)=-0.3320<0$, the state

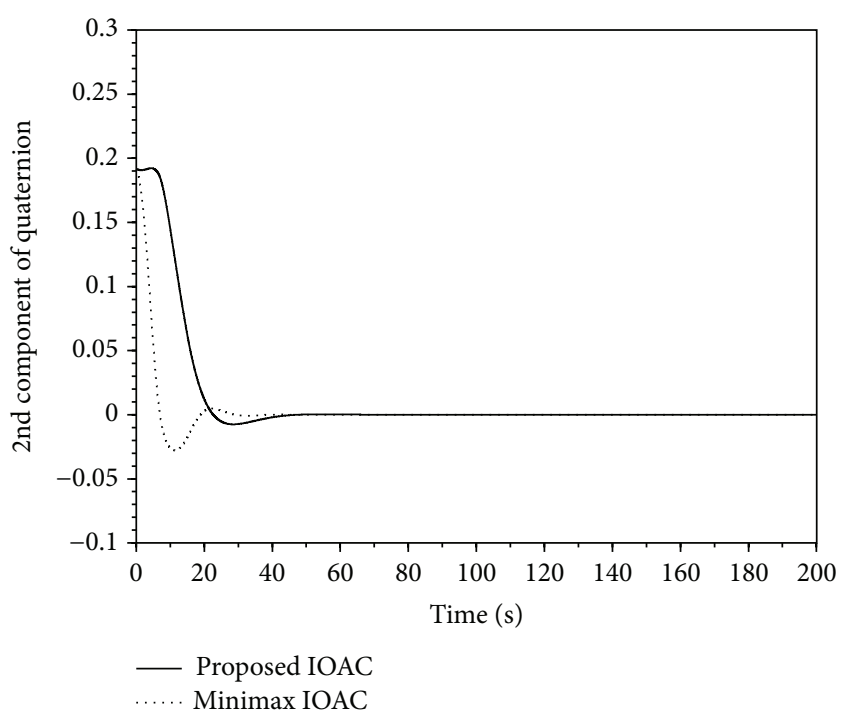

FIgURE 2: Time responses of the 2nd component of quaternion.

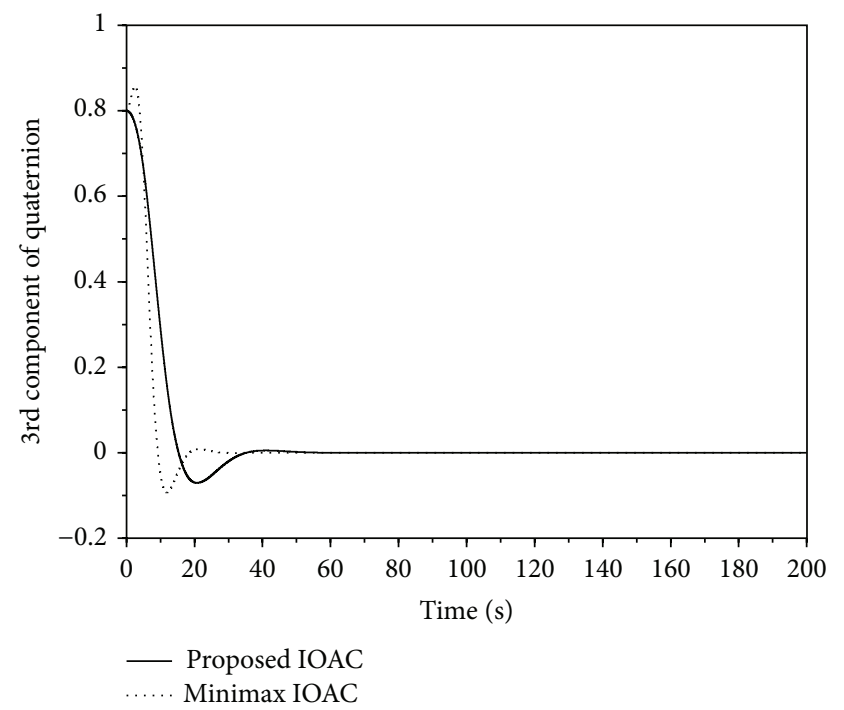

Figure 3: Time responses of the 3rd component of quaternion.

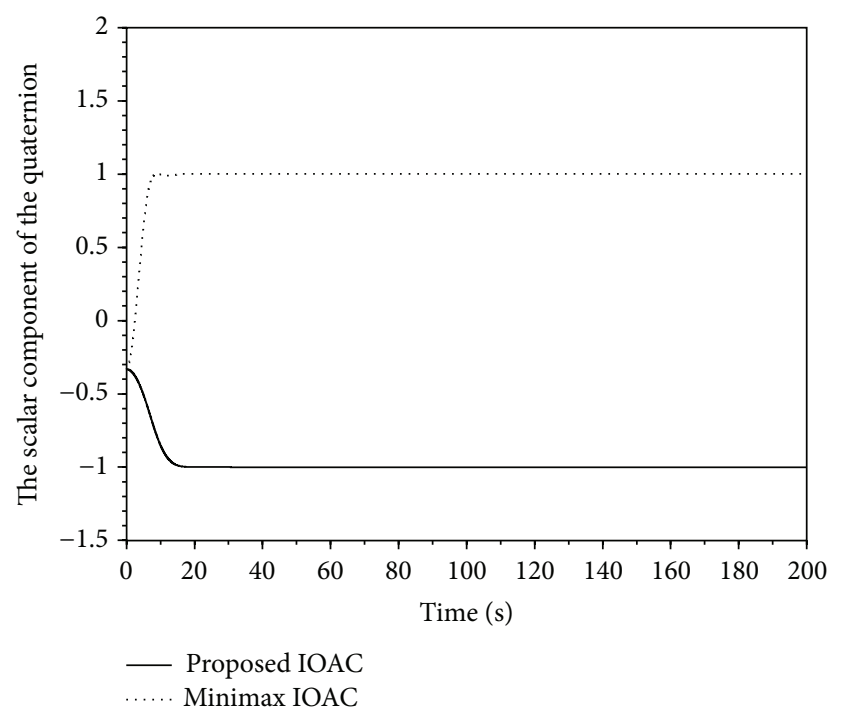

FIGURE 4: Time responses of the scalar of quaternion. 


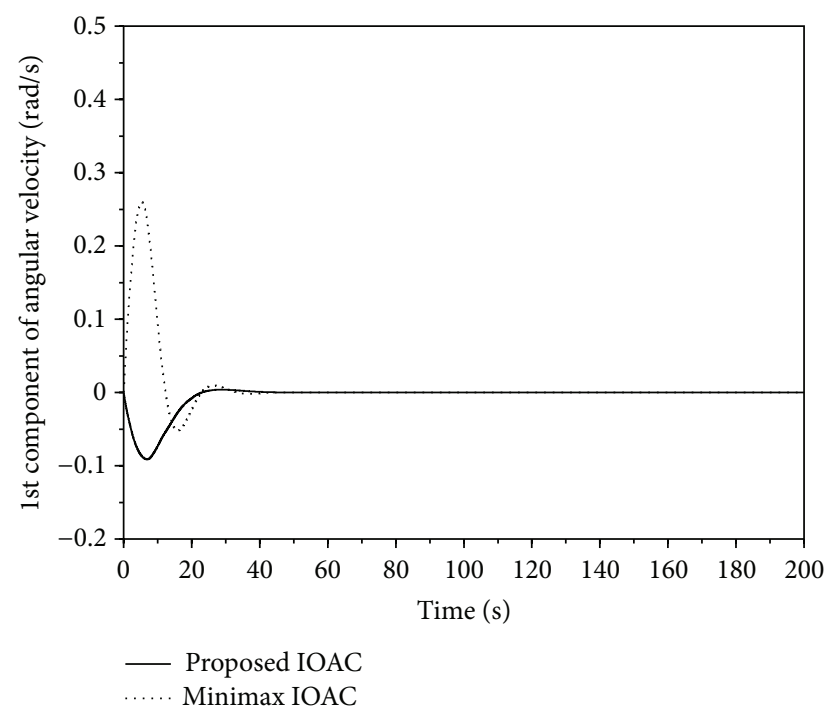

FIGURE 5: Time responses of the 1st component of angular velocities.

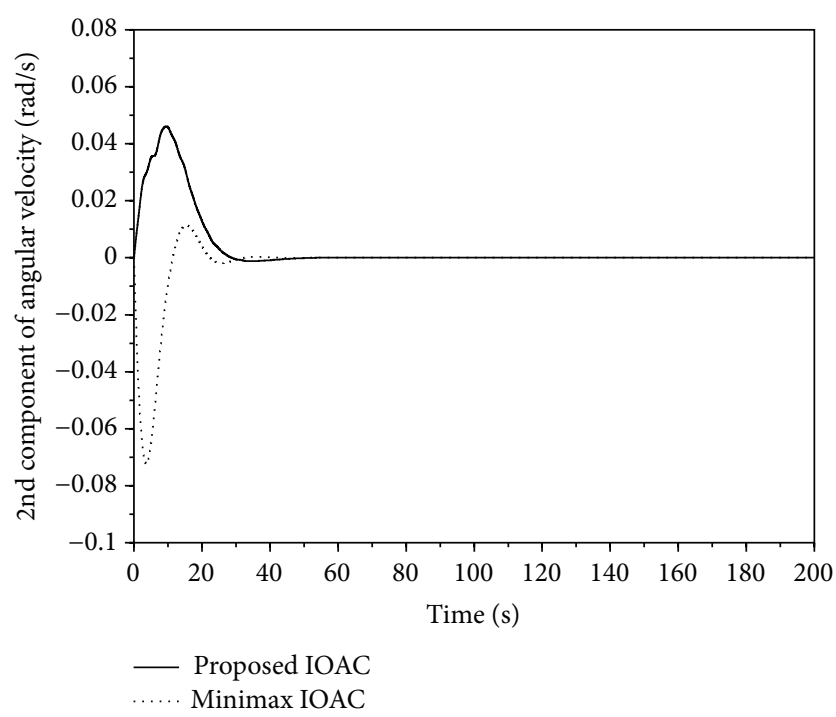

FIGURE 6: Time responses of the 2nd component of angular velocities.

$q_{0}(t)$ should converge to the equilibrium point $q_{0}=-1$. Figure 4 shows that only the proposed controller (72) can force the state $q_{0}(t)$ to the equilibrium point $q_{0}=-1$. Thus, the proposed controller (72) can avoid an unwinding phenomenon, while for the minimax IOAC in [21], an unwinding phenomenon occurs. In Figures 5-7, responses of angular velocities obtained by the proposed controller (72) are smoother. Figures 8-10 show that the minimax IOAC in [21] requires larger magnitude control torques. As shown in Figures 11-14 for minimax IOAC in [21], modal displacements converge to a small region around the zero. However, these good responses of modal displacements are obtained since the minimax IOAC takes large control magnitudes. As shown in Figures 15-17, the proposed SMDO provides fast and accurate estimations of the disturbance vector.

We have made comparisons between the simulation results obtained by minimax IOAC in [21] and proposed

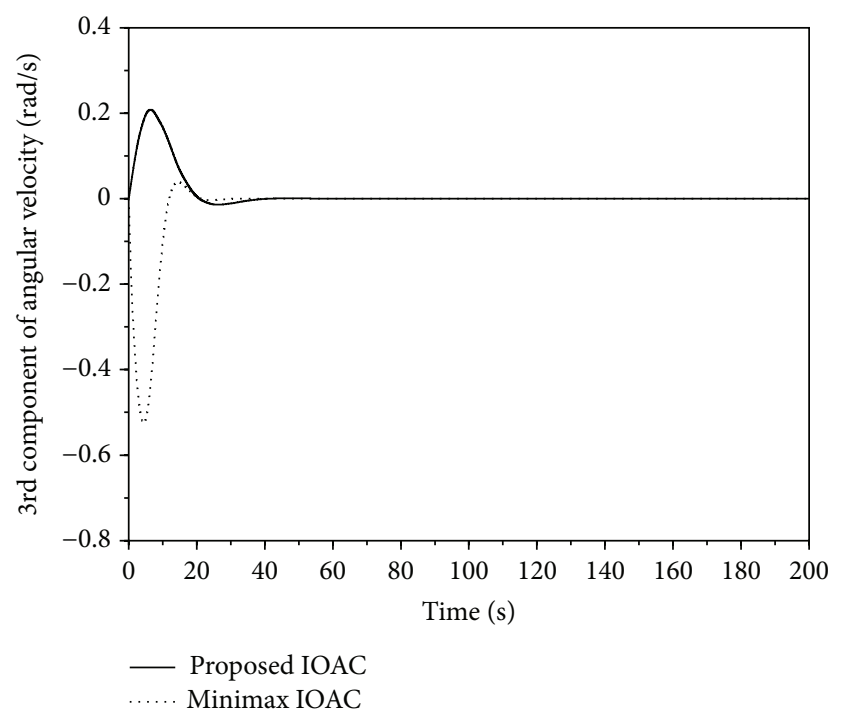

FIGURE 7: Time responses of the 3rd component of angular velocities.

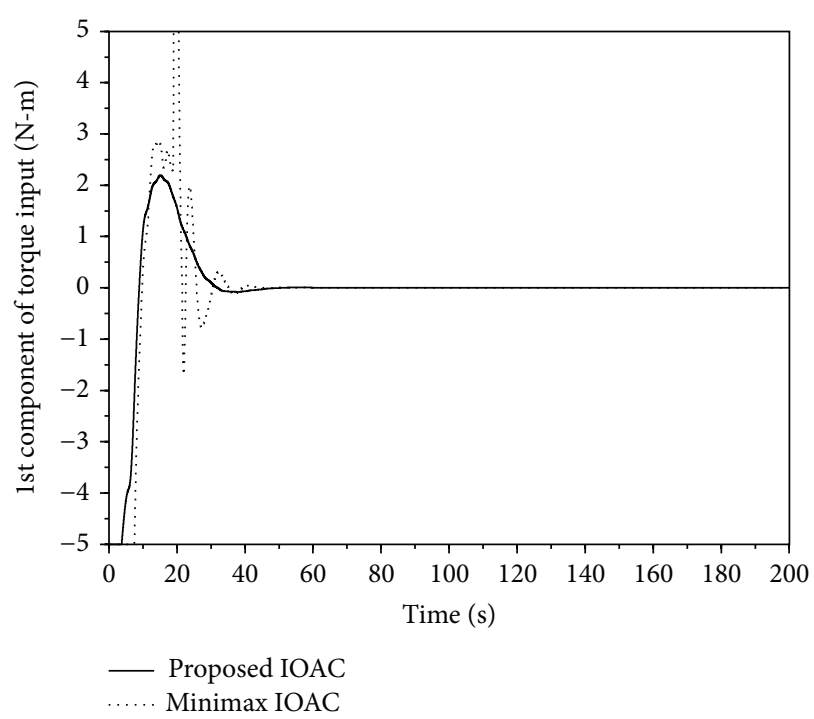

FIgURE 8: Time responses of the 1st component of control torques.

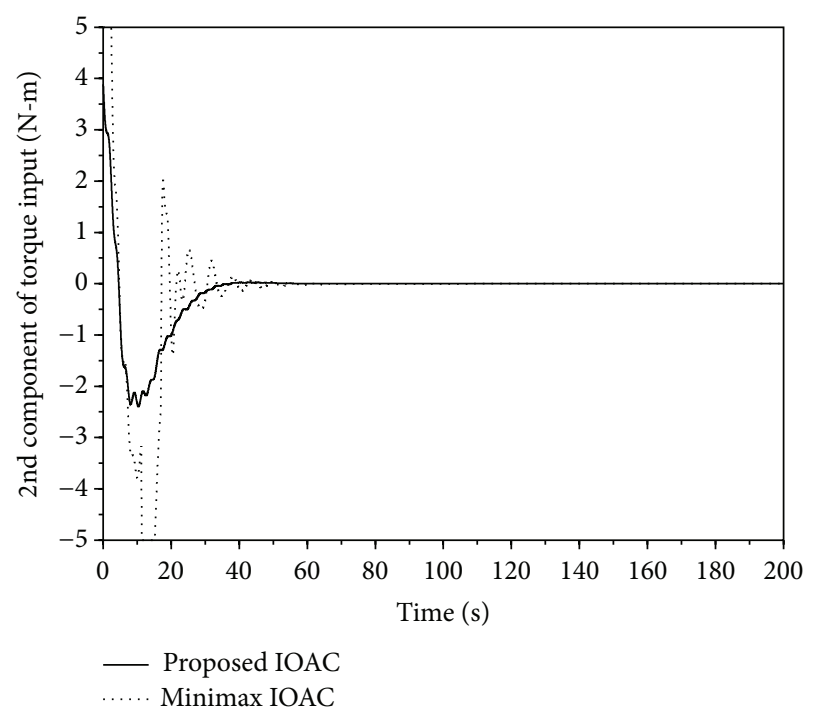

FIGURE 9: Time responses of the 2nd component of control torques. 


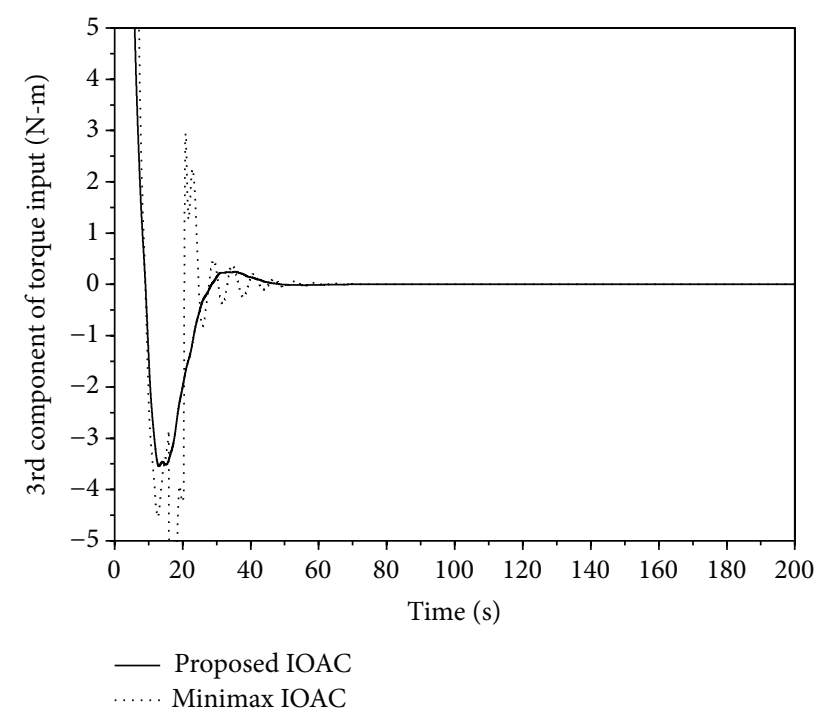

FIGURE 10: Time responses of the 3rd component of control torques.

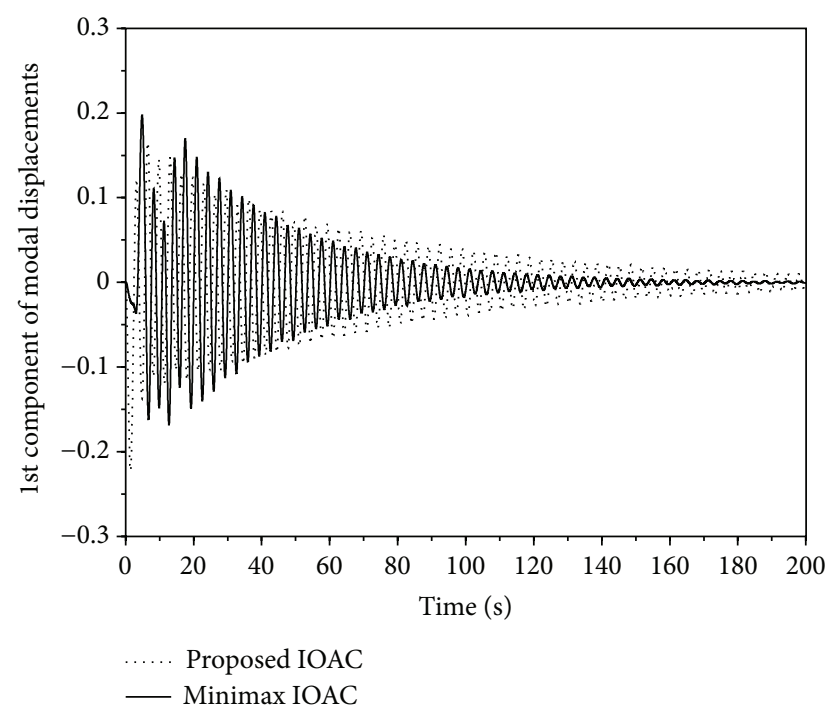

FIGURE 11: Time responses of the 1st component of modal displacements.

IOAC (72). Because both controllers have different cost functionals, they have different magnitudes of control forces and torques and take different convergence speeds to reach the origin. These are not valid enough reasons to find out which one of the two controllers is better. It is found that the proposed control law (72) offers smooth attitude and angular velocity responses and requires smaller magnitudes of control inputs. In view of these simulation results, the proposed IOAC (72) seems to be a more useful control approach for general cases of flexible spacecraft attitude maneuvers. It should be noted that the method of [21] did not consider the optimal control problem of flexible spacecraft, so it is unfair to directly compare this method with our proposed control scheme since our proposed method is designed for the flexible spacecraft system. Moreover, the control performance of the attitude controller proposed in [21] is determined by

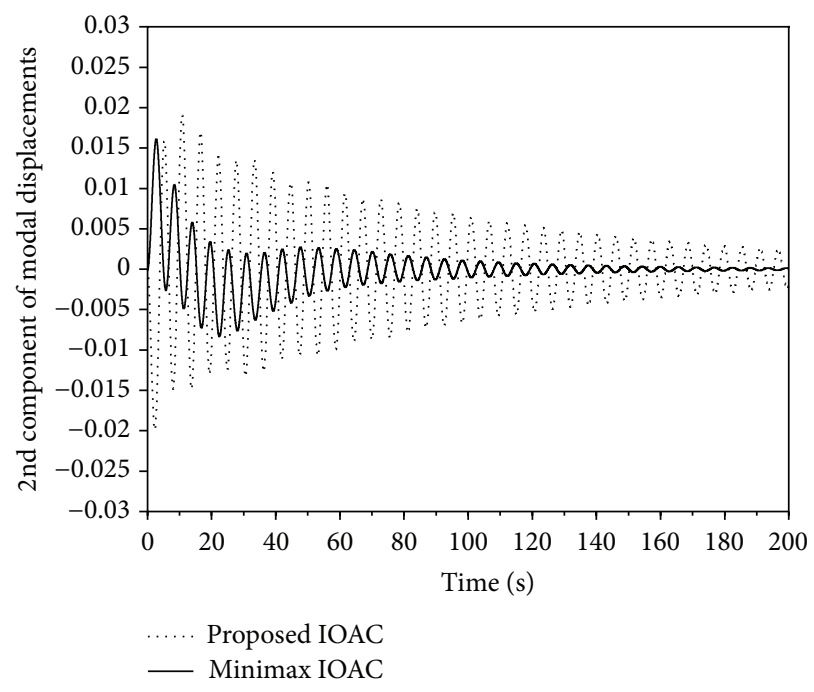

FIGURE 12: Time responses of the 2nd component of modal displacements.

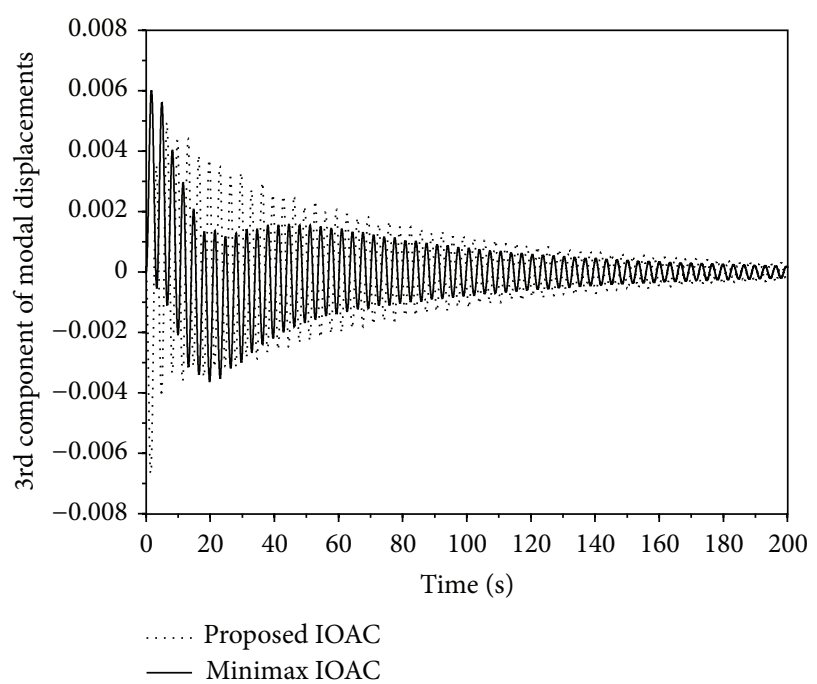

Figure 13: Time responses of the 3rd component of modal displacements.

the control gain parameters, so the control performance of the attitude controller proposed in [21] could give better simulation results by choosing other control gain parameters.

\section{Conclusion}

A robust inverse optimal control scheme of attitude stabilization of a flexible spacecraft in the presence of external disturbances and actuator constraint has been developed. The concepts of inverse optimal control and CLF have been employed to design an inverse optimal attitude control law with actuator saturation. A new SMDO has been designed by using the similar structure to the traditional ESO. The ultimate boundedness of estimation errors has been proven using the Lyapunov technique. It has shown that the developed controller solves the inverse optimal control problem 


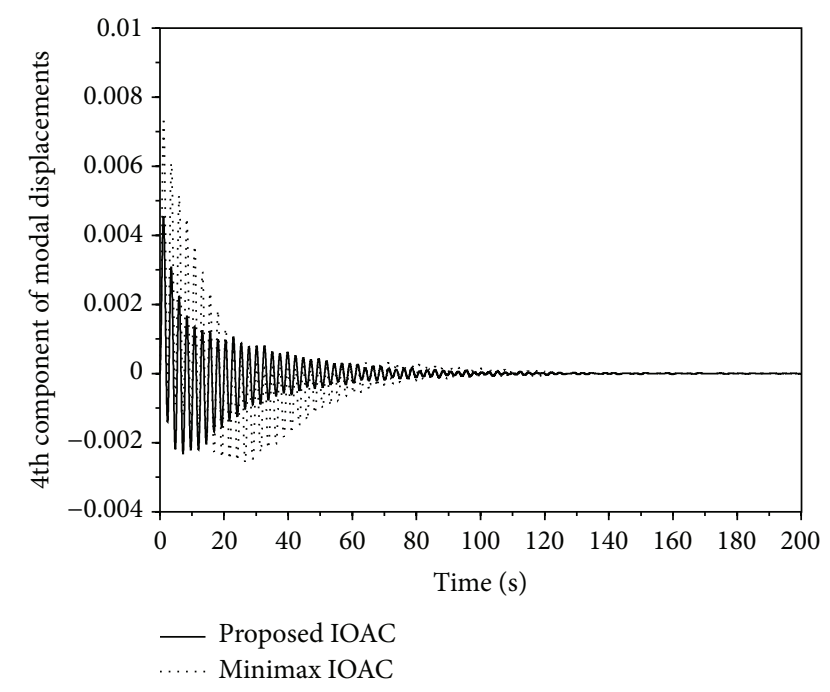

FIGURE 14: Time responses of the 4 th component of modal displacements.

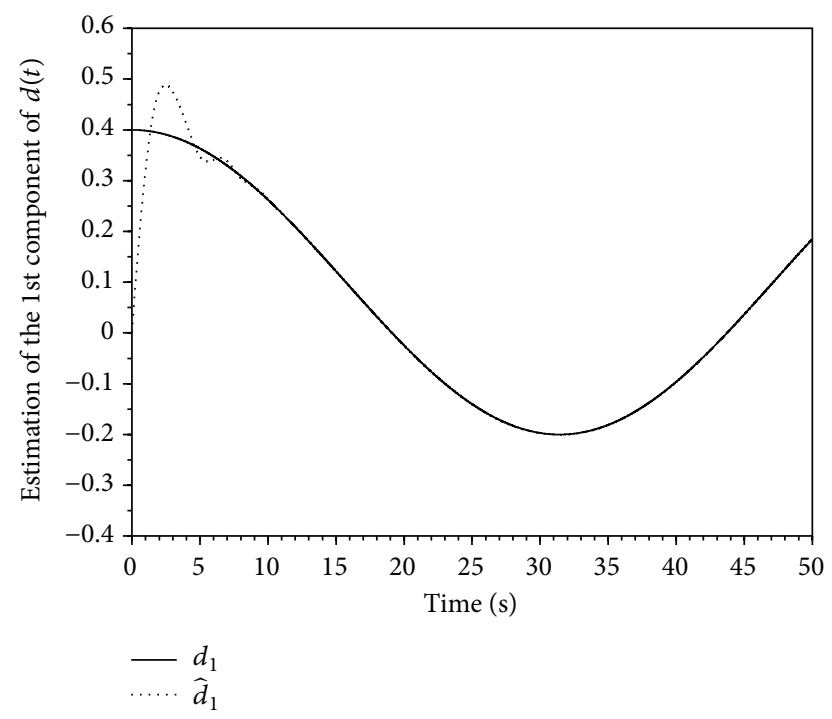

FIGURE 15: Estimation of the 1st component of the disturbance vector.

with input saturation and asymptotically converges to the origin. An example of a rest-to-rest maneuver is presented and simulation results are provided to verify the usefulness of the presented controller.

\section{Competing Interests}

The author declares that there are no competing interests.

\section{Acknowledgments}

The research was supported by King Mongkut's University of Technology North Bangkok, Contract no. KMUTNB-GOV58-13.

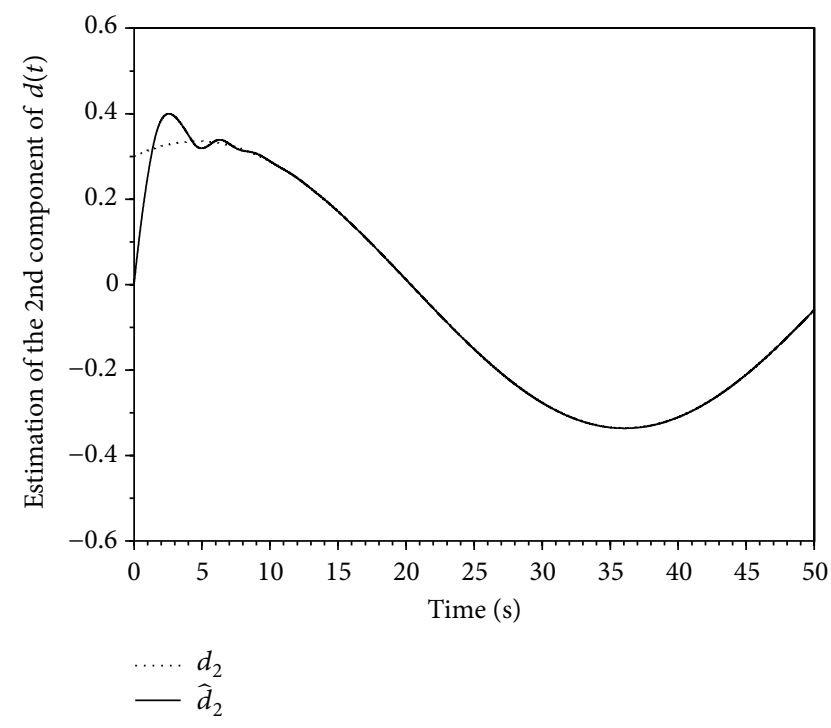

FIGURE 16: Estimation of the 2nd component of the disturbance vector.

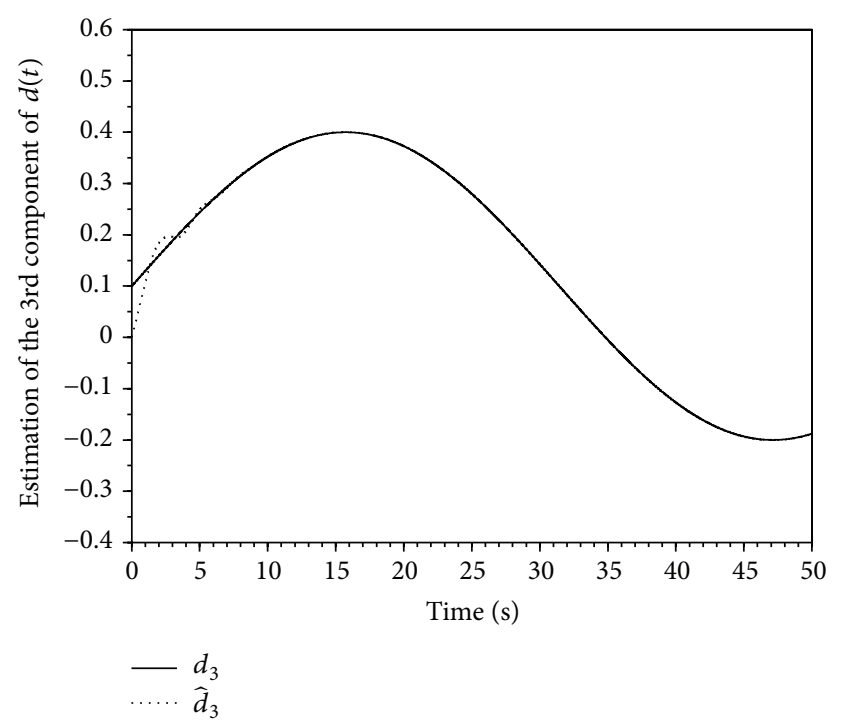

FIGURE 17: Estimation of the 3rd component of the disturbance vector.

\section{References}

[1] S. Di Gennaro, "Passive attitude control of flexible spacecraft from quaternion measurements," Journal of Optimization Theory and Applications, vol. 116, no. 1, pp. 41-60, 2003.

[2] Q.-L. Hu, Z. Wang, and H. Gao, "Sliding mode and shaped input vibration control of flexible systems," IEEE Transactions on Aerospace and Electronic Systems, vol. 44, no. 2, pp. 503-519, 2008.

[3] H. Bang, C.-K. Ha, and J. H. Kim, "Flexible spacecraft attitude maneuver by application of sliding mode control," Acta Astronautica, vol. 57, no. 11, pp. 841-850, 2005.

[4] Q. Hu, "Robust adaptive sliding mode attitude control and vibration damping of flexible spacecraft subject to unknown disturbance and uncertainty," Transactions of the Institute of Measurement and Control, vol. 34, no. 4, pp. 436-447, 2012. 
[5] C. Pukdeboon and A. Jitpattanakul, "Finite-time anti-disturbance inverse optimal attitude tracking control of flexible spacecraft," Mathematical Problems in Engineering, vol. 2013, Article ID 967574, 13 pages, 2013.

[6] C. Zhong, Y. Guo, Z. Yu, L. Wang, and Q. Chen, "Finite-time attitude control for flexible spacecraft with unknown bounded disturbance," Transactions of the Institute of Measurement and Control, vol. 38, no. 2, pp. 240-249, 2016.

[7] J. Zheng, S. P. Banks, and H. Alleyne, "Optimal attitude control for three-axis stabilized flexible spacecraft," Acta Astronautica, vol. 56, no. 5, pp. 519-528, 2005.

[8] D. K. Parrish and D. B. Ridgely, "Attitude control of a satellite using the SDRE method," in Proceedings of the American Control Conference, pp. 942-946, Albuquerque, NM, USA, June 1997.

[9] D. T. Stansbery and J. R. Cloutier, "Position and attitude control of a spacecraft using the state-dependent Riccati equation technique," in Proceedings of the American Control Conference, pp. 1867-1871, Chicago, Ill, USA, June 2000.

[10] M. Xin and S. N. Balakrishnan, "Robust state dependent Riccati equation based attitude control," in Proceedings of the 40th AIAA Aerospace Sciences Meeting and Exhibit, Reno, Nev, USA, 2002.

[11] M. Xin and H. Pan, "Nonlinear optimal control of spacecraft approaching a tumbling target," Aerospace Science and Technology, vol. 15, no. 2, pp. 79-89, 2011.

[12] P. Tsiotras, "Stabilization and optimality results for the attitude control problem," Journal of Guidance, Control, and Dynamics, vol. 19, no. 4, pp. 772-779, 1996.

[13] M. Krstic and P. Tsiotras, "Inverse optimal stabilization of a rigid spacecraft," IEEE Transactions on Automatic Control, vol. 44, no. 5, pp. 1042-1049, 1999.

[14] Y. Park, "Inverse optimal and robust nonlinear attitude control of rigid space-craft," Aerospace Science and Technology, vol. 28, no. 1, pp. 257-265, 2013.

[15] E. D. Sontag, "A universal construction of Artstein's theorem on nonlinear stabilization," Systems and Control Letters, vol. 13, no. 2, pp. 117-123, 1989.

[16] R. A. Freeman and P. V. Kokotović, "Inverse optimality in robust stabilization," SIAM Journal on Control and Optimization, vol. 34, no. 4, pp. 1365-1391, 1996.

[17] R. A. Freeman and P. V. Kokotović, Robust Nonlinear Control Design, Birkh Nauser, Boston, Mass, USA, 1996.

[18] J. A. Primbs, V. Nevistić, and J. C. Doyle, "Nonlinear optimal control: a control Lyapunov function and receding horizon perspective," Asian Journal of Control, vol. 1, no. 1, pp. 14-24, 1999.

[19] W. Kang, "Nonlinear $H_{\infty}$ control and its application to rigid spacecraft," IEEE Transactions on Automatic Control, vol. 40, no. 7, pp. 1281-1285, 1995.

[20] M. Dalsmo and O. Egeland, "Stae feedback $H_{\infty}$-suboptimal control of a rigid spacecraft," IEEE Transactions on Automatic Control, vol. 42, no. 8, pp. 1186-1189, 1997.

[21] Y. Park, "Robust and optimal attitude stabilization of spacecraft with external disturbances," Aerospace Science and Technology, vol. 9, no. 3, pp. 253-259, 2005.

[22] W. Luo, Y.-C. Chu, and K.-V. Ling, "Inverse optimal adaptive control for attitude tracking of spacecraft," IEEE Transactions on Automatic Control, vol. 50, no. 11, pp. 1639-1654, 2005.

[23] Q. Zheng and F. Wu, "Nonlinear $H_{\infty}$ control designs with axisymmetric spacecraft control," Journal of Guidance, Control, and Dynamics, vol. 32, no. 3, pp. 850-859, 2009.
[24] C. Pukdeboon and A. S. Zinober, "Control Lyapunov function optimal sliding mode controllers for attitude tracking of spacecraft," Journal of the Franklin Institute. Engineering and Applied Mathematics, vol. 349, no. 2, pp. 456-475, 2012.

[25] C. Pukdeboon, "Optimal sliding mode controllers for attitude stabilization of flexible spacecraft," Mathematical Problems in Engineering, vol. 2011, Article ID 863092, 20 pages, 2011.

[26] C. G. Mayhew, R. G. Sanfelice, and A. R. Teel, "On path-lifting mechanisms and unwinding in quaternion-based attitude control," IEEE Transactions on Automatic Control, vol. 58, no. 5, pp. 1179-1191, 2013.

[27] J. R. Wertz, Spacecraft Attitude Determination and Control, Kluwer Academic, Dordrecht, Netherlands, 1978.

[28] S. Di Gennaro, "Output stabilization of flexible spacecraft with active vibration suppression," IEEE Transactions on Aerospace and Electronic Systems, vol. 39, no. 3, pp. 747-759, 2003.

[29] P. Kokotović and M. Arcak, "Constructive nonlinear control: a historical perspective," Automatica, vol. 37, no. 5, pp. 637-662, 2001.

[30] M. D. Shuster, "A survey of attitude representations," Journal of the Astronautical Sciences, vol. 41, no. 4, pp. 439-517, 1993.

[31] J. Erdong and S. Zhaowei, "Passivity-based control for a flexible spacecraft in the presence of disturbances," International Journal of Non-Linear Mechanics, vol. 45, no. 4, pp. 348-356, 2010.

[32] J. Han, "From PID to active disturbance rejection control," IEEE Transactions on Industrial Electronics, vol. 56, no. 3, pp. 900906, 2009.

[33] C. Pukdeboon, "Inverse optimal sliding mode control of spacecraft with coupled translation and attitude dynamics," International Journal of Systems Science, vol. 46, no. 13, pp. 24212438, 2015.

[34] S. Li, X. Yang, and D. Yang, "Active disturbance rejection control for high pointing accuracy and rotation speed," Automatica, vol. 45, no. 8, pp. 1854-1860, 2009. 


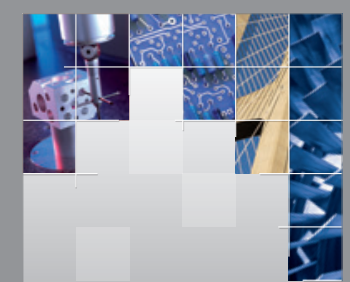

\section{Enfincering}
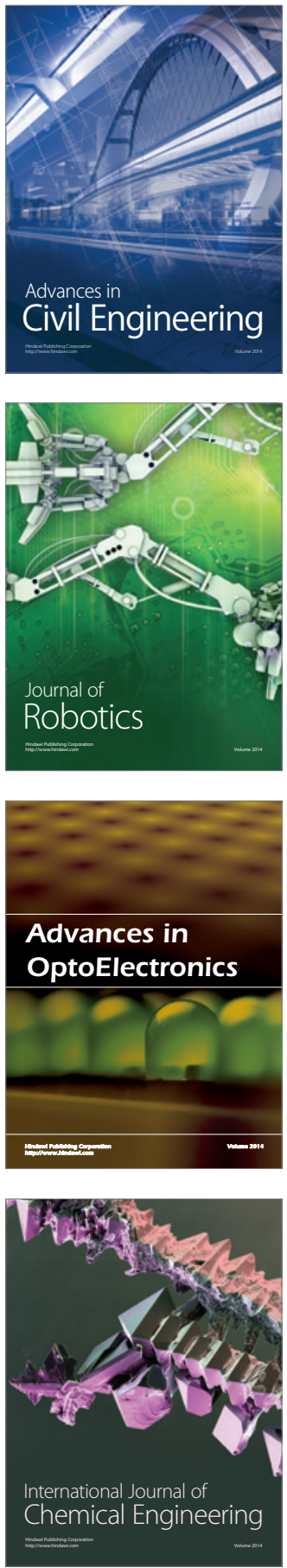

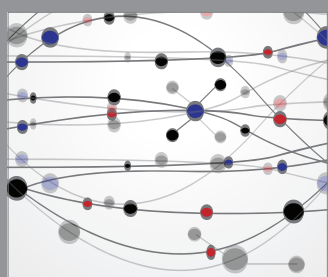

The Scientific World Journal

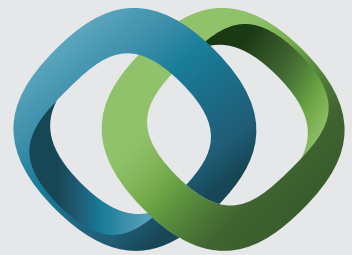

\section{Hindawi}

Submit your manuscripts at

http://www.hindawi.com
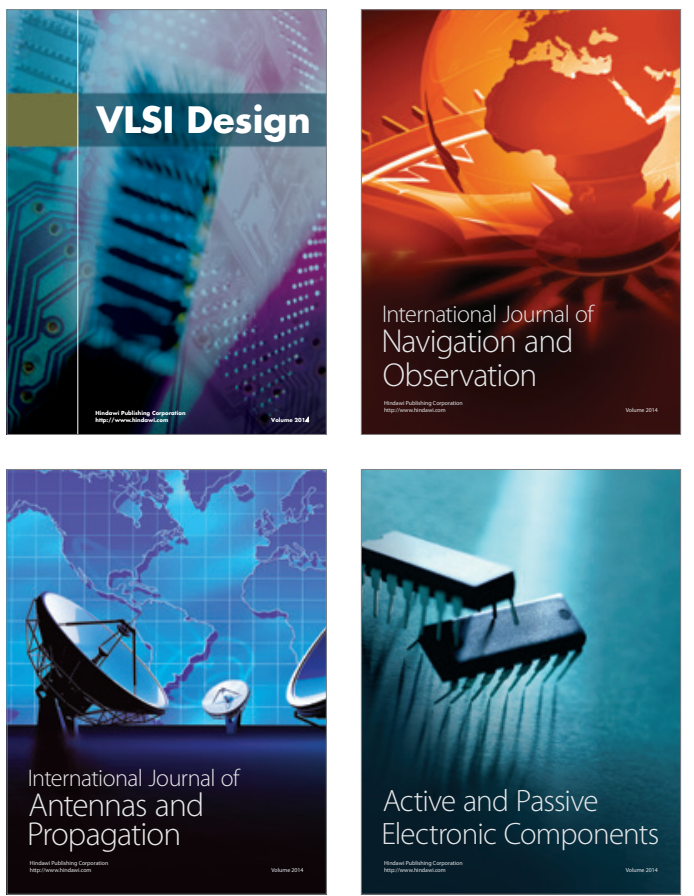
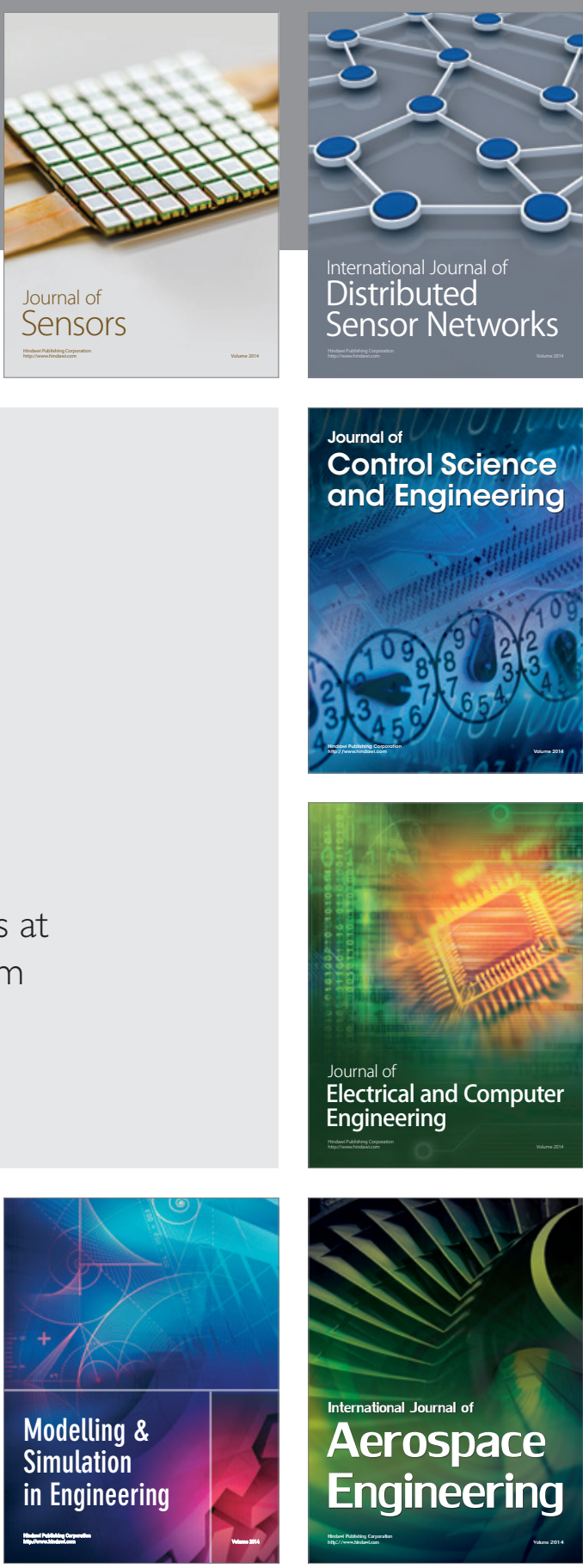

International Journal of

Distributed

Sensor Networks

Journal of

Control Science

and Engineering
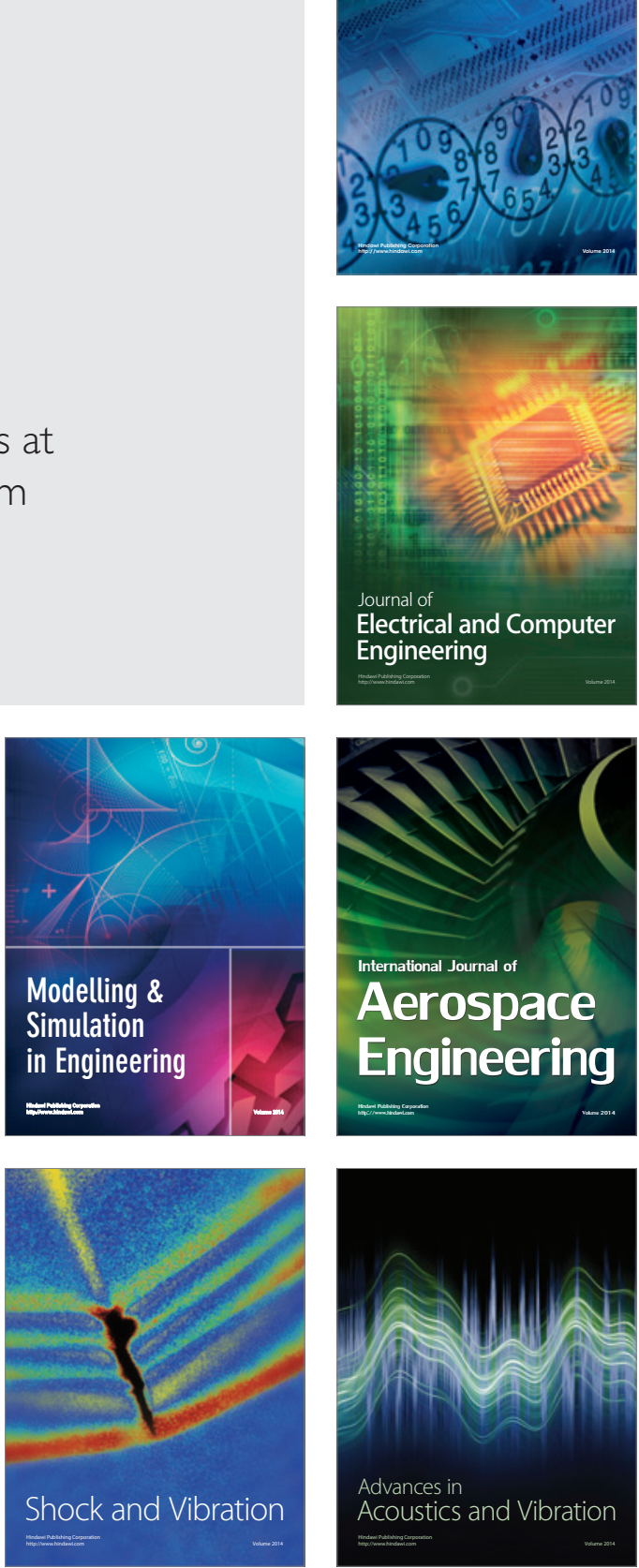